$\underline{\text { Preprint typeset in JHEP style - HYPER VERSION }}$

\title{
Relations of loop partial amplitudes in gauge theory by Unitarity cut method
}

\author{
Bo Feng ${ }^{a b c}$, Yin $\operatorname{Jia}^{a}$, Rijun Huang ${ }^{a *}$ \\ ${ }^{a}$ Zhejiang Institute of Modern Physics, Zhejiang University, Hangzhou, 310027, P. R. China \\ ${ }^{b}$ Center of Mathematical Science, Zhejiang University, Hangzhou, China \\ ${ }^{c}$ Kavli Institute for Theoretical Physics China, CAS, Beijing 100190, China
}

\begin{abstract}
It is well known that under the color-decomposition, one-loop amplitude of gluons contains partial amplitudes of single and double trace structures, and particularly all partial amplitudes of double trace structure can be expressed as a linear combination of partial amplitudes of single trace structure. Using unitarity cut method, we prove that this result is the natural consequence of tree-level Kleiss-Kuijf relation. Generalizing the unitarity cut method to two-loop (triple cut in this case), we show that, unlike the one-loop case, partial amplitudes of double and triple trace structures can not be expressed as a linear combination of partial amplitudes of leading-color single trace structure. For partial amplitudes of subleading-color single trace structure, we have shown a very nontrivial Kleiss-Kuijf relation for six and seven-point amplitudes, which is one new result of our paper and can not be obtained by $U(1)$-decoupling method. Mysteriously, when we consider the case of eight points, Kleiss-Kuijf relation must be modified for subleading-color single trace partial amplitudes.
\end{abstract}

${ }^{*}$ The unusual ordering of authors is just to satisfy outdated requirement for $\mathrm{Ph}$. Degree. 


\section{Contents}

$\begin{array}{ll}\text { 1. Introduction } & 1\end{array}$

2. Partial amplitudes of one loop amplitude 4

2.1 Revisit of $U(1)$-decoupling method

2.1.1 The general $U(1)$-decoupling equations

2.1.2 Analysis of six-point amplitude 6

2.2 Unitarity cut method 8

2.2.1 The example of four-point amplitude 10

2.2 .2 Proof of general case 14

3. Partial amplitudes of two-loop amplitude $\quad 19$

3.1 Understanding four-point amplitude from $U(1)$-decoupling method 21

3.2 Further understanding of four-point amplitude from unitarity cut method 23

3.3 KK-like relation for partial amplitudes of subleading-color single trace 26

4. Conclusion $\quad 30$

A. Direct verification of relations for two-loop four-point amplitude 31

B. The proof of identity $A_{1,1,2}(1 ; 2 ; 3,4)=A_{2,1,1}(1,2 ; 3 ; 4) \quad 32$

\section{Introduction}

Great effects have been made to explore the hidden simplicity of amplitudes in recent years. Different from traditional lagrangian description, new approaches, such as the unitarity cut method [1, 2, 3, 4, 5, 6], the Cachazo-Svrcek-Witten (CSW) rule [7] based on the twistor string theory proposed by Witten [8] and the Britto-Cachazo-Feng-Witten (BCFW) on-shell recursion relation [9, 10], have shown their advantages in the calculation of scattering amplitudes. These new methods are not only useful for calculation, but also for understanding many properties of amplitudes.

For example, the well known color-reflection, $U(1)$-decoupling [11, 12, 13] and Kleiss-Kuijf (KK) relation [14 $]^{1}$, together with the newly discovered Bern-Carrasco-Johansson (BCJ) relation [18 for colorordered tree-level partial amplitudes of gauge theory, have been re-understood from the point of view of

\footnotetext{
${ }^{1}$ The KK relation has been proved by field theory method in 15 and by string theory method in [16, 17.
} 
pure field theory in [19, 20] (See further generalization and discussion [21, 22, 23]). The tree-level partial amplitudes are defined by the color-decomposition of full tree-level amplitude based on its color trace structure $T^{a}$ [1], 24, 25, 26] (or structure constant $f^{a b c}$ [15]) as follows

$$
\mathcal{A}_{n}^{\text {full }}\left(\left\{k_{i}, \lambda_{i}, a_{i}\right\}\right)=g^{n-2} \sum_{\sigma \in S_{n} / Z_{n}} \operatorname{Tr}\left(T^{a_{\sigma_{1}}} \ldots T^{a_{\sigma_{n}}}\right) A_{n}\left(k_{\sigma_{1}}^{\lambda_{\sigma_{1}}}, \ldots, k_{\sigma_{n}}^{\lambda_{\sigma_{n}}}\right),
$$

where $k_{i}, \lambda_{i}, a_{i}$ are respectively momentum, helicity and color index of $i$-th external gluon, and $S_{n} / Z_{n}$ represents the permutations on $n$-particles up to cyclic ordering. The decomposition (1.1) has separated the dynamical information (given by the partial amplitudes) from the group information (given by trace structure).

For tree-level partial amplitudes $A_{n}$ defined in (1.1), because the cyclic invariant of trace, there are $(n-1)$ ! partial amplitudes. However not all of these partial amplitudes are algebraic independent and they are related to each other by cross symmetry and other considerations. First there is nontrivial KK relation [14] given by

$$
A_{n}(1, \alpha, n, \beta)=(-)^{n_{\beta}} \sum_{\sigma \in O P\{\alpha\} \cup\left\{\beta^{T}\right\}} A_{n}(1, \sigma, n),
$$

where $n_{\beta}$ is the number of $\beta$-set and the Order-Preserved(OP) sum is over all permutations of the set $\alpha \bigcup \beta^{T}$ where the relative ordering in each set $\alpha$ and $\beta^{T}$ (which is the reversed ordering of set $\beta$ ) is preserved $^{2}$. The KK relation will reduce the number of independent partial amplitudes to $(n-2)$ !. Beyond the KK relation, there is also BCJ relation [18], which further reduces the number of independent partial amplitudes to $(n-3)$ !, where kinematic factors $s_{i j}=\left(k_{i}+k_{j}\right)^{2}$ show up in the coefficients of the relation. The BCJ relation was originally observed from non-trivial Jacobi relations between $s, t, u$-channels of Feynman diagrams, and then proved as the imaginary part of monodromy relations in string theory [16, 17]. This relation has been proved by BCFW recursion relation in pure field theory [19, 20] ${ }^{3}$.

Beyond tree-level amplitude, a similar color decomposition can be introduced [12, 3]. One loop amplitude can be decomposed into partial amplitudes with single trace $N_{c} \operatorname{Tr}(X)$ structure and double trace $\operatorname{Tr}(X) \operatorname{Tr}(Y)$ structure, while two loop amplitude can be decomposed into partial amplitudes with leadingcolor single trace $N_{c}^{2} \operatorname{Tr}(X)$, subleading-color single trace $\operatorname{Tr}(X)$, double trace $N_{c} \operatorname{Tr}(X) \operatorname{Tr}(Y)$ and triple trace $\operatorname{Tr}(X) \operatorname{Tr}(Y) \operatorname{Tr}(Z)$ structures. For general $L$-loop amplitude, there are at most $(L+1)$ traces appearing with planar or non-planar structures [12].

As the case of tree-level, not all loop partial amplitudes are independent to each other. A traditional method to analyze their relation is to use $U(1)$-decoupling equations based on the observation that photon

\footnotetext{
${ }^{2}$ One non-trivial example of KK relation with six gluons is given as follows

$$
\begin{aligned}
A(1,2,3,6,4,5)= & A(1,2,3,5,4,6)+A(1,2,5,3,4,6)+A(1,2,5,4,3,6) \\
& +A(1,5,4,2,3,6)+A(1,5,2,4,3,6)+A(1,5,2,3,4,6) .
\end{aligned}
$$
}

\footnotetext{
${ }^{3}$ An extension of BCJ relation to matter fields can be found in [27. Other related works see [28, 29, 30, 31, 32].
} 
will decouple from theory where there is no matter field [12]. The steps to get these relations are as follows. First we take one generator to be $U(1)$, then color traces will reorganize themselves. Because the full amplitude vanishes and the reorganized color traces are independent to each other, the corresponding coefficient of each reduced color trace will be zero too. Thus we obtain a series of equations among partial amplitudes, which are called $U(1)$-decoupling equations. By solving these equations, we could express some partial amplitudes by other partial amplitudes. However, we know that there are relations that can not be discovered by $U(1)$-decoupling method as given in [3] for one-loop partial amplitudes, where string inspired arguments have to be used. Are there other useful methods, besides $U(1)$-decoupling method, for loop partial amplitudes?

To answer the question, first we notice that loop-level partial amplitudes can also be studied by direct calculations using, for example, Feynman diagrams or other methods. Among these methods, unitarity cut method [1, 2, 3, 5, 6] and generalized unitarity cut method [4, 33, 34, 35, 36] (i.e, the leading singularity method) have been proved to be particularly useful to obtain loop results (especially one-loop results) from tree-level input ${ }^{4}$.

Encouraged by the success of unitarity cut method in the calculation of one-loop scattering amplitudes, we find that just like BCFW recursion relation has been used to prove relations between tree-level partial amplitudes, unitarity cut method can also been used to understand relations between loop-level partial amplitudes. In other words, besides the $U(1)$-decoupling method, there is indeed another method available to our investigation.

The skeleton of this paper is as follows. In section two we study one-loop amplitude from both $U(1)$ decoupling and unitarity cut method. Especially we have reproved the KK-like relation (2.2) for one-loop partial amplitudes using the unitarity cut method. In section three, two-loop amplitude is investigated similarly, where possible KK relation for subleading-color single trace partial amplitudes is discussed for six, seven and eight points. In the last section, a summary is given, as well as some comments on possible future directions. Some calculation details and checking are given in two Appendixes.

Notation: For simplicity, in this paper we will consider the $U(N)$ gauge group instead of $S U(N)$ gauge group. The $U(N)$ generators are a set of hermitian $N \times N$ matrices with normalization $\operatorname{Tr}\left(T^{a} T^{b}\right)=\delta^{a b}$, and the structure constant is defined as

$$
\left[T^{a}, T^{b}\right]=i f^{a b c} T^{c}
$$

Thus the Fierz identities of $U(N)$ gauge theory are

$$
\sum_{a} \operatorname{Tr}\left(T^{a} X\right) \operatorname{Tr}\left(T^{a} Y\right)=\operatorname{Tr}(X Y), \quad \sum_{a} \operatorname{Tr}\left(T^{a} X T^{a} Y\right)=\operatorname{Tr}(X) \operatorname{Tr}(Y),
$$

where one special case is

$$
\sum_{a} \operatorname{Tr}\left(X T^{a} T^{a} Y\right)=N_{c} \operatorname{Tr}(X Y) .
$$

These relations are useful when we discuss the color structure using the unitarity cut method.

\footnotetext{
${ }^{4}$ BCJ-like relation in loop level has also been investigated in several works, see 32, 37, 38, 39, 40.
} 


\section{Partial amplitudes of one loop amplitude}

The color decomposition of loop amplitude in $U(N)$ gauge theory can be understood from view of $U(N)$ open string, whose infinite-tension limit reduces to gauge theory [12]. One can also sketch the various trace structures of color decomposition from arguments based on Feynman diagram analysis. Different from tree amplitudes, double trace structure appears in one-loop level, and the corresponding partial amplitudes can be expressed as linear combination of primitive (partial) amplitudes, i.e., amplitudes of single trace structure. Schematically, the color decomposition of $n$-point one-loop amplitude for $U(N)$ gauge theory can be written as [3]

$$
\mathcal{A}_{n}^{\text {full }}\left(\left\{k_{i}, \lambda_{i}, a_{i}\right\}\right)=\sum_{J} n_{J} \sum_{m=0}^{\lfloor n / 2\rfloor} \sum_{\sigma \in S_{n} / S_{n ; m}} \operatorname{Gr}_{n-m, m}(\sigma) A_{n-m, m}^{[J]}\left(\sigma_{1}, \sigma_{2}, \ldots, \sigma_{n-m} ; \sigma_{n-m+1}, \ldots, \sigma_{n}\right),
$$

where $\lfloor x\rfloor$ is the largest integer less than or equal to $x$ and $n_{J}$ is the number of particles of spin $J$. The color structure for primitive amplitude is (For convenience we abbreviate $\operatorname{Tr}\left(T^{a_{1}} \cdots T^{a_{n}}\right)$ as $\operatorname{Tr}\left(a_{1}, \cdots, a_{n}\right)$ )

$$
\operatorname{Gr}_{n, 0}=N_{c} \operatorname{Tr}\left(a_{1}, \cdots, a_{n}\right)
$$

and for other partial amplitudes is

$$
\mathrm{Gr}_{n-m, m}=\operatorname{Tr}\left(a_{1}, \cdots, n-m\right) \operatorname{Tr}(n-m+1, \cdots, n) .
$$

$S_{n}$ is the set of all permutations of $n$ objects, and $S_{n ; m}$ is the subset leaving $\mathrm{Gr}_{n-m, m}$ invariant. If the gauge group is $S U(N)$, then there is no $\mathrm{Gr}_{n-1,1}$ term since $\operatorname{Tr}\left(T_{a}\right)=0$. However, the partial amplitude $A_{n-1,1}$ is well defined and non-zero. To make $A_{n-1,1}$ explicit in the expression, we consider $U(N)$ gauge theory instead of $S U(N)$.

It is found that the partial amplitudes $A_{n-m, m}$ of double trace structure $\operatorname{Gr}_{n-m, m}(m \neq 0)$ have algebraic relation with primitive amplitudes $A_{n, 0}$ of single trace, i.e., $A_{n-m, m}$ can be expressed as linear combination of $A_{n, 0}$, thus the computing of primitive amplitudes is enough for constructing full one-loop amplitude. The relation is given by [3]

$$
A_{n-m, m}\left(\alpha_{1}, \alpha_{2}, \ldots, \alpha_{n-m} ; \beta_{1}, \ldots, \beta_{m}\right)=(-1)^{m} \sum_{\sigma \in C O P\{\alpha\} \bigcup\left\{\beta^{T}\right\}} A_{n, 0}(\sigma)
$$

where $\beta^{T}$ is the set of $\beta$ with reversed ordering, and $C O P\{\alpha\} \bigcup\left\{\beta^{T}\right\}$ is the set of all permutations of $\left\{\alpha, \beta^{T}\right\}$ preserving the cyclic ordering inside the set $\alpha$ and $\beta^{T}$, but allowing all possible relative orderings between two sets $\alpha$ and $\beta^{T}$. This equation can be expected from analysis of open string amplitude [12] or from the view of new color-decomposition discussed in [15]. The aim of this section is to understand (2.2) using unitarity cut method.

Before going to details, let us give some remarks. As shown in [15], the (2.2) is the direct consequence of color Jacobi structure at one-loop level. The Jaboci structure is easily seen from $f^{a b c}$ but not so transparent 
for color-ordered partial amplitudes at tree and loop levels. For example, Jacobi structure of tree-level color-ordered amplitude is hidden implicitly in the KK relation and BCJ relation. Our discussions in this section will provide another point of view to understand the same question, although our method is a little bit circuitous.

\subsection{Revisit of $U(1)$-decoupling method}

Before discussing the unitarity cut method, let us revisit the $U(1)$-decoupling equation carefully. We will show that with the $U(1)$-decoupling equation, a relation such like (2.2) will not emerge. Thus new thought is needed to understand (2.2).

\subsubsection{The general $U(1)$-decoupling equations}

The central idea of $U(1)$-decoupling equation is to choose one of generators to be $U(1)$, then the original trace structure 2.1) will reorganize itself to new color trace structure. Since photon does not interact with others, coefficients of new color traces will be zero.

To demonstrate, let us consider four-point amplitude which has three kinds of partial amplitudes: one with single trace structure $A_{4,0}$ and the other two with double trace structure, $A_{3,1}$ and $A_{2,2}$. Their corresponding color structures can be abbreviated as $(4 \mid 0),(3 \mid 1)$ and $(2 \mid 2)$. By setting one generator as identity, these color structures reduce to $(3 \mid 0)$ and $(2 \mid 1)$ as follows

$$
(4 \mid 0) \rightarrow(3 \mid 0) \quad, \quad(3 \mid 1) \rightarrow(2 \mid 1) \text { or }(3 \mid 0), \quad(2 \mid 2) \rightarrow(2 \mid 1) .
$$

More explicitly, with $T_{4}$ being $U(1)$, the reduced color structure $(3 \mid 0)=N_{c} \operatorname{Tr}(1,2,3)$ gives the following $U(1)$-decoupling equation

$$
A_{4,0}(1,2,3,4)+A_{4,0}(1,2,4,3)+A_{4,0}(1,4,2,3)+A_{3,1}(1,2,3 ; 4)=0
$$

while the reduced color structure $(2 \mid 1)=\operatorname{Tr}(1,2) \operatorname{Tr}(3)$ gives (there are other $(2 \mid 1)$ trace structures)

$$
A_{3,1}(1,2,4 ; 3)+A_{3,1}(1,4,2 ; 3)+A_{2,2}(1,2 ; 3,4)=0 .
$$

Using the equation (2.4) we can solve $A_{3,1}$ as a linear combination of $A_{4,0}$. Then together with (2.5), we can finally solve $A_{2,2}$ as a linear combination of $A_{4,0}$.

For general $n$, with trace structure (where we have put trace structure implicitly as parameters of partial amplitudes $A$ )

$$
\mathcal{A}^{1-\text { loop }}=\sum N_{c} A_{n, 0}\left(\sigma_{1}, \ldots, \sigma_{n}\right)+\sum_{m} A_{n-m, m}\left(\sigma_{1}, \ldots, \sigma_{n-m} ; \beta_{1}, \ldots, \beta_{n}\right)
$$

when we take, for example, $T^{n}$ to be $U(1)$, then $(n-m \mid m)$ structure will reduce to either $(n-m \mid m-1)$ or $(n-m-1 \mid m)$ structures depending on where $T^{n}$ locates. Collecting all terms having the same reduced color structure we get one $U(1)$-decoupling equation. By taking different reduced color structures and different $T^{a}$ to be $U(1)$ we can get different equations. 
We can go further by taking more than one $T^{a}$ to be $U(1)$. However, since after one $U(1)$ reduction, all coefficients of reduced color structures are zero already, taking more than one generator to be $U(1)$ does not give new relations. Thus to get all independent $U(1)$-decoupling equations, we just need to take one $T^{a}$ to be $U(1)$ with $a=1, \ldots, n$ and write down all equations obtained by this way.

Having above general discussions, now let us write down equations obtained by taking $T^{n}$ to be $U(1)$. The reduced trace structure $\operatorname{Tr}(1, \ldots, m-1) \operatorname{Tr}(m, \ldots, n-1)$ will receive contributions from partial amplitudes of original trace structures $(n-m \mid m)$ and $(n-m+1 \mid m-1)$, so the corresponding $U(1)-$ decoupling equation is

$0=\sum_{\sigma \in \text { cyclic }} A_{n-m, m}\left(\sigma_{1}, \ldots, \sigma_{m-1}, n ; m, \ldots, n-1\right)+\sum_{\sigma \in \text { cyclic }} A_{n-m+1, m-1}\left(1, \ldots, m-1 ; \sigma_{m}, \ldots, \sigma_{n-1}, n\right)$

where $1 \leq m \leq\lfloor n / 2\rfloor$. When $m=1$, (2.7) can be used to solve $A_{n-1,1}$ by single trace part $A_{n, 0}$ as

$$
A_{n-1,1}(1, \ldots, n-1 ; n)=-\sum_{\sigma \in \text { cyclic }} A_{n, 0}\left(\sigma_{1}, \ldots, \sigma_{n-1}, n\right)
$$

When $m=2$, 2.7) contains only one $A_{n-2,2}$ thus we can solve it as

$$
\begin{aligned}
A_{n-2,2}(1, \ldots, n-2 ; n-1, n) & =-\sum_{\sigma \in \text { cyclic }} A_{n-1,1}\left(\sigma_{1}, \ldots, \sigma_{n-2}, n ; n-1\right) \\
& =\sum_{\sigma \in \text { cyclic } \alpha \in \text { cyclic }} \sum_{n \in C} A_{n, 0}\left(\alpha_{\sigma_{1}}, \ldots, \alpha_{\sigma_{n-2}}, \alpha_{n}, n-1\right) \\
& =\sum_{\sigma \in C O P\{1, \ldots, n-2\} \cup\{n, n-1\}} A_{n, 0}(\sigma) .
\end{aligned}
$$

Things become more complicated when $m \geq 3$. The reason is that amplitudes $A_{n-m, m}$ always appear in group in (2.7) and there is no way to separate them. As we will see explicitly in six-point example, $U(1)$-decoupling equations are not enough to solve $A_{n-m, m}$ by $A_{n, 0}$ as given in (2.2), but they do give hints.

\subsubsection{Analysis of six-point amplitude}

As we have mentioned, when $m \geq 3$, it is impossible to solve all $A_{n-m, m}$ by $A_{n, 0}$ directly through $U(1)$ decoupling equations. To see it clearly, we take the simplest example where the phenomenon happens, i.e., the one-loop six-point amplitude. First we write down $U(1)$-decoupling relation for $n=6$ explicitly [12] as

$$
\begin{aligned}
0 & =\left[A_{5,1}\left(\sigma_{1}, \ldots, \sigma_{5} ; 6\right)+\sum_{\text {cyclic }} A_{6,0}\left(6, \sigma_{1}, \ldots, \sigma_{5}\right)\right], \\
0 & =\sum_{\text {cyclic } \sigma} A_{5,1}\left(6, \sigma_{1}, \ldots, \sigma_{4} ; \beta_{1}\right)+A_{4,2}\left(\sigma_{1}, \ldots, \sigma_{4} ; 6, \beta_{1}\right), \\
0 & =\sum_{\text {cyclic } \sigma} A_{4,2}\left(6, \sigma_{1}, \ldots, \sigma_{3} ; \beta_{1}, \beta_{2}\right)+\sum_{\text {cyclic } \beta} A_{3,3}\left(\sigma_{1}, \ldots, \sigma_{3} ; 6, \beta_{1}, \beta_{2}\right),
\end{aligned}
$$


where $T^{6}$ has been set as $U(1)$ identity. Using (2.10) we can solve any $A_{5,1}$ by partial amplitudes $A_{6,0}$ :

$$
\begin{aligned}
A_{5,1}(1,2,3,4,5 ; 6)= & -A_{6}(6,1,2,3,4,5)-A_{6}(6,5,1,2,3,4)-A_{6}(6,4,5,1,2,3) \\
& -A_{6}(6,3,4,5,1,2)-A_{6}(6,2,3,4,5,1) .
\end{aligned}
$$

Having the $A_{5,1}$ we can use (2.11) to solve $A_{4,2}$ by partial amplitudes $A_{6,0}$ as

$$
A_{4,2}(1,2,3,4 ; 5,6)=\sum_{\sigma \in C O P\{1,2,3,4\} \cup\{6,5\}} A_{6,0}(\sigma) .
$$

The tricky part is $A_{3,3}$. From equation 2.12 we have

$$
\begin{aligned}
& A_{3,3}(1,2,3 ; 6,4,5)+A_{3,3}(1,2,3 ; 6,5,4)=X_{1}=-\sum_{\sigma \in \text { cyclic }} A_{4,2}\left(6, \sigma_{1}, \sigma_{2}, \sigma_{3} ; 4,5\right), \\
& A_{3,3}(1,3,2 ; 6,4,5)+A_{3,3}(1,3,2 ; 6,5,4)=X_{2}=-\sum_{\sigma \in \text { cyclic }} A_{4,2}\left(6, \sigma_{1}, \sigma_{3}, \sigma_{2} ; 4,5\right),
\end{aligned}
$$

where $X_{1}, X_{2}$ are, in fact, a linear combination of $A_{6,0}$. Taking leg 5 or 4 to be photon we can obtain other two equations, which are similar to (2.15) and (2.16) by relabeling $\{4,5,6\} \rightarrow\{4,6,5\}$ and $\{4,5,6\} \rightarrow$ $\{6,4,5\}$. However, the left hand sides of (2.15) and (2.16) are invariant directly under above relabeling, while the invariant of the right hand sides can be seen explicitly only when expanded as a linear combination of $A_{6,0}$. Thus there are no new relations coming from taking $T^{4}, T^{5}$ to be $U(1)$.

Using $T_{1}$ to be $U(1)$ we get another equations

$$
\begin{aligned}
& A_{3,3}(1,2,3 ; 4,5,6)+A_{3,3}(1,3,2 ; 4,5,6)=\widetilde{X}_{1}=-\sum_{\sigma \in \text { cyclic }} A_{4,2}\left(1, \sigma_{4}, \sigma_{5}, \sigma_{6} ; 2,3\right), \\
& A_{3,3}(1,2,3 ; 4,6,5)+A_{3,3}(1,3,2 ; 4,6,5)=\widetilde{X}_{2}=-\sum_{\sigma \in \text { cyclic }} A_{4,2}\left(1, \sigma_{4}, \sigma_{6}, \sigma_{5} ; 2,3\right) .
\end{aligned}
$$

Similarly it can be shown that taking $T^{2}, T^{3}$ to be $U(1)$ will not give new relations.

Equations (2.15), 2.16), (2.17) and (2.18) are all independent ones we can obtain, provided that the legs $1,2,3$ in $A_{3,3}$ are all in one trace and the others are all in another trace. There are four partial amplitudes $t_{1}=A_{3,3}(1,2,3 ; 4,5,6), t_{2}=A_{3,3}(1,3,2 ; 4,5,6), t_{3}=A_{3,3}(1,2,3 ; 4,6,5), t_{4}=A_{3,3}(1,3,2 ; 4,6,5)$, and four equations, which, when written in matrix form, are

$$
\left(\begin{array}{c}
X_{1} \\
X_{2} \\
\widetilde{X}_{1} \\
\widetilde{X}_{2}
\end{array}\right)=\left(\begin{array}{llll}
1 & 0 & 1 & 0 \\
0 & 1 & 0 & 1 \\
1 & 1 & 0 & 0 \\
0 & 0 & 1 & 1
\end{array}\right)\left(\begin{array}{c}
t_{1} \\
t_{2} \\
t_{3} \\
t_{4}
\end{array}\right) .
$$

This one has unique solution when and only when the determinant is non-zero. However, it is easy to check that the determinant is indeed zero and we find following solution:

$$
t_{1}=t_{4}+\widetilde{X}_{1}-X_{2}, \quad t_{2}=-t_{4}+X_{2}, \quad t_{3}=-t_{4}-\widetilde{X}_{1}+X_{1}+X_{2}
$$


which indicates the impossibility of expressing all partial amplitudes $A_{3,3}$ as a linear combination of $A_{6,0}$ from $U(1)$-decoupling equations ${ }^{5}$.

Although $U(1)$-decoupling equations can not solve $A_{3,3}$ as combinations of $A_{6,0}$, they may give some hints to the solution (2.2). The key is to write the $X_{i}, \tilde{X}_{i}$ as

$$
X_{1}=(456) \bigcup(123)+(465) \bigcup(123), \quad X_{2}=(456) \bigcup(132)+(465) \bigcup(132),
$$

and

$$
\tilde{X}_{1}=(123) \bigcup(456)+(132) \bigcup(456), \quad \widetilde{X}_{2}=(123) \bigcup(465)+(132) \bigcup(465),
$$

where we have simplified $\sum_{\sigma \in C O P\{1,2,3\} \bigcup\{4,5,6\}} A(\sigma)$ as $(456) \bigcup(123)$. With these rewriting, it is very natural to make some identifications. From (2.15) and (2.16) there are two choices can be made. The choice $(\mathrm{A})$ is given by

$$
\begin{aligned}
& A_{3,3}(1,2,3 ; 4,5,6)=(1,2,3) \bigcup(4,5,6), \quad A_{3,3}(1,2,3 ; 6,5,4)=(1,2,3) \bigcup(6,5,4), \\
& A_{3,3}(1,3,2 ; 4,5,6)=(1,3,2) \bigcup(4,5,6), \quad A_{3,3}(1,3,2 ; 6,5,4)=(1,3,2) \bigcup(6,5,4),
\end{aligned}
$$

while the choice $(\mathrm{B})$ is given by

$$
\begin{aligned}
& A_{3,3}(1,2,3 ; 4,5,6)=(1,2,3) \bigcup(6,5,4), \quad A_{3,3}(1,2,3 ; 6,5,4)=(1,2,3) \bigcup(4,5,6), \\
& A_{3,3}(1,3,2 ; 4,5,6)=(1,3,2) \bigcup(6,5,4), \quad A_{3,3}(1,3,2 ; 6,5,4)=(1,3,2) \bigcup(4,5,6) .
\end{aligned}
$$

Both choices are consistent with (2.17) and (2.18) if we notice that the color-order reversed relation means $(\alpha \bigcup \beta)=(-)^{n}\left(\alpha^{T} \bigcup \beta^{T}\right)$, i.e., $(1,2,3) \cup(4,5,6)=(1,3,2) \bigcup(6,5,4)$. However, the right solution is the choice (B). Our six-point example may be too special and when we move to higher points, hints will be more explicit.

\subsection{Unitarity cut method}

Unitarity cut method has been proved to be very useful for calculations of loop amplitudes. For one-loop amplitude, Passarino-Veltman reduction shows that any one-loop amplitude can be expanded to some known basis with rational coefficients [41]. The basis contains scalar integrals with topologies as tadpole, bubble, triangle and box in pure 4D theory (in this case we need to add rational remaining functions) or in general $(4-2 \epsilon)$-dimension with pentagon ${ }^{6}$. Thus loop calculations are reduced to finding coefficients of corresponding basis [2]. The advantage of unitarity cut method for calculations is that inputs are on-shell tree-level amplitudes which have all desired symmetries, including gauge symmetry.

\footnotetext{
${ }^{5}$ The consistent condition requires $\widetilde{X}_{1}+\widetilde{X}_{2}-X_{1}-X_{2}=0$, which can be verified trivially by writing $X_{i}, \widetilde{X}_{i}$ as sum of $A_{6,0}$.

${ }^{6}$ There are some recent works on the basis of two-loop amplitudes, for example see [49]. The basis for general multiloop amplitudes is not clear yet, but we can still get useful information for analyzing by using unitarity cut method.
} 
Much calculations that have been done using unitarity cut method are for color-ordered partial amplitudes. However, as we will show in this subsection, unitarity cut method is also very useful for calculations of whole amplitudes as well as the understanding of color structure of general loop amplitudes.

To start, let us write down the expression for calculating the whole one-loop amplitude at a given cut channel with momentum $K^{7}$

$$
\left.\mathcal{A}_{n}^{1-\text { loop }}\right|_{\text {cut }}=\sum_{\text {states of } \ell_{1}, \ell_{2}} \mathcal{A}_{L}^{\text {full tree }}\left(\ell_{1}, \alpha_{L}, \ell_{2}, \beta_{L}\right) \mathcal{A}_{R}^{\text {full tree }}\left(-\ell_{1}, \alpha_{R},-\ell_{2}, \beta_{R}\right),
$$

where the ordering of $\left\{\ell_{1}, \alpha_{L}, \ell_{2}, \beta_{L}\right\}$ does not mean anything because the input is the full on-shell tree amplitude $\mathcal{A}$. To uniquely determine the full one-loop amplitude, we need to calculate all possible cut channels in unitarity cut method. A few of remarks are in order before we go on. First we have used double cuts, which can not access tadpole coefficients. Fortunately, for gauge theory which is massless, there is no tadpole contribution. Secondly, we require that there should be at least two external legs in $A_{L}$ or $A_{R}$ for each cut channel, which is satisfied for massless particles. Thirdly, to get complete result for one-loop amplitude using unitarity cut method, the cut momenta $\ell_{1}, \ell_{2}$ should be in general $(4-2 \epsilon)$ dimension. Thus we have assumed properties which we will use later, such as the KK relation, will hold in general D-dimension. This is true for gauge theory because as we have remarked, KK relation is a pure group theory relation.

With above clarifications, we will discuss how various trace structures of one-loop amplitude, i.e., the single trace and double trace structures, show up in the unitarity cut method. Substituting two full tree amplitudes with their color-decompositions in (2.25), and noticing that the sum over states of $\ell_{1}, \ell_{2}$ include the sum over colors, we have (where for simplicity we have written $\ell_{1}$ for $T^{\ell_{1}}$ )

$$
\sum_{\ell_{2}} \sum_{\ell_{1}} \operatorname{Tr}\left(\ell_{1}, \alpha_{L}, \ell_{2}, \beta_{L}\right) \operatorname{Tr}\left(\ell_{1}, \alpha_{R}, \ell_{2}, \beta_{R}\right)=\sum_{\ell_{2}} \operatorname{Tr}\left(\alpha_{L}, \ell_{2}, \beta_{L}, \alpha_{R}, \ell_{2}, \beta_{R}\right)=\operatorname{Tr}\left(\beta_{L}, \alpha_{R}\right) \operatorname{Tr}\left(\beta_{R}, \alpha_{L}\right)
$$

where (1.5) has been used. Equation (2.26) is the general double trace color structure and when we set $\left\{\alpha_{L}, \beta_{R}\right\}$ or $\left\{\beta_{L}, \alpha_{R}\right\}$ empty, it is reduced to the single trace structure $N_{c} \operatorname{Tr}\left(\beta_{L}, \alpha_{R}\right)$ or $N_{c} \operatorname{Tr}\left(\alpha_{L}, \beta_{R}\right)$. Correspondingly, coefficients for the double trace structure $\operatorname{Tr}\left(\alpha_{L}, \beta_{R}\right) \operatorname{Tr}\left(\beta_{L}, \alpha_{R}\right)$ will have contributions from following cut-input

$$
\sum_{h_{1}, h_{2}} A_{L}\left(\ell_{1}^{h_{1}}, \alpha_{L}, \ell_{2}^{h_{2}}, \beta_{L}\right) A_{R}\left(-\ell_{1}^{-h_{1}}, \alpha_{R},-\ell_{2}^{-h_{2}}, \beta_{R}\right)
$$

where $A_{L}, A_{R}$ are color-ordered tree-level partial amplitudes. For the same trace structure, another cutinput,

$$
\sum_{h_{1}, h_{2}} A_{L}\left(\ell_{1}^{h_{1}}, \beta_{L}, \ell_{2}^{h_{2}}, \alpha_{L}\right) A_{R}\left(-\ell_{1}^{-h_{1}}, \beta_{R},-\ell_{2}^{-h_{2}}, \alpha_{R}\right)
$$

\footnotetext{
${ }^{7}$ Similar idea has been discussed in paper [4, 45], where the unitarity cut method plus the CSW rule has been applied to the one-loop MHV amplitudes.
} 
also gives contribution, which comes from exchanging set $\alpha$ and $\beta$, or equivalently, exchanging $\ell_{1}, \ell_{2}$. In principle we should sum up these two contributions together. However, for one-loop, it can be seen that above two inputs (2.27) and (2.28) give the same contributions. Using this freedom we can write 2.25) formally as

$$
\begin{aligned}
\left.\mathcal{A}_{n}^{1-\text { loop }}\right|_{\text {cut }}= & \sum_{L, R}\left[\sum_{P\left\{\alpha_{L}(1), \ell_{2}, \beta_{L}\right\}} \operatorname{Tr}\left(\ell_{1}, \alpha_{L}(1), \ell_{2}, \beta_{L}\right) A_{L}\left(\ell_{1}, \alpha_{L}(1), \ell_{2}, \beta_{L}\right)\right. \\
& \left.\times \sum_{P\left\{\alpha_{R}, \ell_{2}, \beta_{R}\right\}} \operatorname{Tr}\left(\ell_{1}, \alpha_{R}, \ell_{2}, \beta_{R}\right) A_{R}\left(-\ell_{1}, \alpha_{R},-\ell_{2}, \beta_{R}\right)\right] \\
& +\left\{\ell_{1} \leftrightarrow \ell_{2}\right\}
\end{aligned}
$$

where $\alpha_{L}(1)$ means that particle 1 belongs to set $\alpha_{L}$ and the sum $\sum_{L, R}$ means we need to consider all allowed double cuts. The whole amplitude $\mathcal{A}_{n}^{1-\text { loop }}$ is not equal to the right hand side of $(2.29)$ but is determined by the right hand side through the unitarity cut method. Also as we have remarked, since all calculations are the same after exchanging $\ell_{1}, \ell_{2}$ we could only consider the first term of (2.29).

Tree-level partial amplitudes in (2.29) are with all possible color-orderings, but by using $U(1)$-decoupling relation, KK relation and BCJ relation among them, we may establish relations between different trace structures. More explicitly, assuming there is a combination of one-loop partial amplitudes of different trace structures

$$
I=\sum_{i} c_{i} A_{n-i, i}
$$

if under all possible double cuts, above combination is zero, i.e., $\sum_{i} c_{i} A_{L, i} A_{R, i}=0$, then we can claim that the combination $I$ is zero, i.e., there is a nontrivial relation among these one-loop partial amplitudes, up to three points discussed below equation (2.25).

With these general discussions, now we will apply the unitarity cut method to investigate relations among one-loop partial amplitudes. We will study the four-point amplitude in detail as an example and then give a proof of result (2.2).

\subsubsection{The example of four-point amplitude}

As an illustration of unitarity cut method, let us give a detail analysis of four-point one-loop amplitude. After fixing leg 1 in the left tree amplitudes (we can always do that), and decomposing the full tree 
amplitude as partial amplitudes, (2.25) becomes

$$
\begin{aligned}
\left.\mathcal{A}_{4}^{1-\text { loop }}\right|_{\text {cut }}= & \sum_{P\left\{\ell_{2}, 1,2\right\}} \operatorname{Tr}\left(\ell_{1}, \ell_{2}, 1,2\right) A_{L}\left(\ell_{1}, \ell_{2}, 1,2\right) \sum_{P\left\{-\ell_{2}, 3,4\right\}} \operatorname{Tr}\left(\ell_{1}, \ell_{2}, 3,4\right) A_{R}\left(-\ell_{1},-\ell_{2}, 3,4\right) \\
& +\sum_{P\left\{\ell_{2}, 1,3\right\}} \operatorname{Tr}\left(\ell_{1}, \ell_{2}, 1,3\right) A_{L}\left(\ell_{1}, \ell_{2}, 1,3\right) \sum_{P\left\{-\ell_{2}, 2,4\right\}} \operatorname{Tr}\left(\ell_{1}, \ell_{2}, 2,4\right) A_{R}\left(-\ell_{1},-\ell_{2}, 2,4\right) \\
& +\sum_{P\left\{\ell_{2}, 1,4\right\}} \operatorname{Tr}\left(\ell_{1}, \ell_{2}, 1,4\right) A_{L}\left(\ell_{1}, \ell_{2}, 1,4\right) \sum_{P\left\{-\ell_{2}, 2,3\right\}} \operatorname{Tr}\left(\ell_{1}, \ell_{2}, 2,3\right) A_{R}\left(-\ell_{1},-\ell_{2}, 2,3\right),
\end{aligned}
$$

where using cyclic symmetry we can always fix $\ell_{1}$ at the first position and $P\{\alpha\}$ means all permutations on set $\{\alpha\}$. There are totally $3 \times 3 ! \times 3 !=108$ terms in the right hand side of (2.31), and they contribute to different trace structures. These terms can be written down in the form (2.29) as

$$
\begin{aligned}
\left.\mathcal{A}_{4}^{1-\text { loop }}\right|_{\text {cut }}= & \left(\operatorname{Tr}\left(\ell_{1}, 1, \ell_{2}, 2\right) A_{L}\left(\ell_{1}, 1, \ell_{2}, 2\right)+\operatorname{Tr}\left(\ell_{1}, 1,2, \ell_{2}\right) A_{L}\left(\ell_{1}, 1,2, \ell_{2}\right)\right. \\
& \left.+\operatorname{Tr}\left(\ell_{1}, 2,1, \ell_{2}\right) A_{L}\left(\ell_{1}, 2,1, \ell_{2}\right)\right) \times \sum_{P\left\{-\ell_{2}, 3,4\right\}} \operatorname{Tr}\left(\ell_{1}, \ell_{2}, 3,4\right) A_{R}\left(-\ell_{1},-\ell_{2}, 3,4\right) \\
& +\left(\operatorname{Tr}\left(\ell_{1}, 1, \ell_{2}, 3\right) A_{L}\left(\ell_{1}, 1, \ell_{2}, 3\right)+\operatorname{Tr}\left(\ell_{1}, 1,3, \ell_{2}\right) A_{L}\left(\ell_{1}, 1,3, \ell_{2}\right)\right. \\
& \left.+\operatorname{Tr}\left(\ell_{1}, 3,1, \ell_{2}\right) A_{L}\left(\ell_{1}, 3,1, \ell_{2}\right)\right) \times \sum_{P\left\{-\ell_{2}, 2,4\right\}} \operatorname{Tr}\left(\ell_{1}, \ell_{2}, 2,4\right) A_{R}\left(-\ell_{1},-\ell_{2}, 2,4\right) \\
& \left(\operatorname{Tr}\left(\ell_{1}, 1, \ell_{2}, 4\right) A_{L}\left(\ell_{1}, 1, \ell_{2}, 4\right)+\operatorname{Tr}\left(\ell_{1}, 1,4, \ell_{2}\right) A_{L}\left(\ell_{1}, 1,4, \ell_{2}\right)\right. \\
& \left.+\operatorname{Tr}\left(\ell_{1}, 4,1, \ell_{2}\right) A_{L}\left(\ell_{1}, 4,1, \ell_{2}\right)\right) \times \sum_{P\left\{-\ell_{2}, 2,3\right\}} \operatorname{Tr}\left(\ell_{1}, \ell_{2}, 2,3\right) A_{R}\left(-\ell_{1},-\ell_{2}, 2,3\right) \\
+ & \left\{\ell_{1} \leftrightarrow \ell_{2}\right\} .
\end{aligned}
$$

Now let us consider contributions to various trace structures using (2.32).

Firstly we consider the single trace structure. For example, the single trace $N_{c} \operatorname{Tr}(1,2,3,4)$ comes from four terms:

$$
\begin{aligned}
& \operatorname{Tr}\left(\ell_{1}, 1,2, \ell_{2}\right) \operatorname{Tr}\left(\ell_{1}, \ell_{2}, 3,4\right) A_{L}\left(\ell_{1}, 1,2, \ell_{2}\right) A_{R}\left(-\ell_{1},-\ell_{2}, 3,4\right), \\
& \operatorname{Tr}\left(\ell_{1}, 4,1, \ell_{2}\right) \operatorname{Tr}\left(\ell_{1}, \ell_{2}, 2,3\right) A_{L}\left(\ell_{1}, 4,1, \ell_{2}\right) A_{R}\left(-\ell_{1},-\ell_{2}, 2,3\right),
\end{aligned}
$$

and other two terms with $\left\{\ell_{1} \leftrightarrow \ell_{2}\right\}$. Thus we can write

$$
\left.A_{4,0}(1,2,3,4)\right|_{\text {cut }}=A_{L}\left(\ell_{1}, 1,2, \ell_{2}\right) A_{R}\left(-\ell_{1},-\ell_{2}, 3,4\right)+A_{L}\left(\ell_{1}, 4,1, \ell_{2}\right) A_{R}\left(-\ell_{1},-\ell_{2}, 2,3\right)+\left\{\ell_{1} \leftrightarrow \ell_{2}\right\}(2.3
$$

For the color ordering $(1,2,3,4)$ we can determine the amplitude by considering two double cuts: cut $K_{12}$ and cut $K_{41}$, and they are exactly the two terms we have written down in (2.33). 
Next we consider double trace structure. For $A_{3,1}(1,2,3 ; 4)$ with trace structure $\operatorname{Tr}(1,2,3) \operatorname{Tr}(4)$, terms that contribute to this trace structure are

$$
\begin{aligned}
& \operatorname{Tr}\left(\ell_{1}, 1,2, \ell_{2}\right) \operatorname{Tr}\left(\ell_{1}, 4, \ell_{2}, 3\right) A_{L}\left(\ell_{1}, 1,2, \ell_{2}\right) A_{R}\left(-\ell_{1}, 4,-\ell_{2}, 3\right), \\
& \operatorname{Tr}\left(\ell_{1}, 3,1, \ell_{2}\right) \operatorname{Tr}\left(\ell_{1}, 4, \ell_{2}, 2\right) A_{L}\left(\ell_{1}, 3,1, \ell_{2}\right) A_{R}\left(-\ell_{1}, 4,-\ell_{2}, 2\right), \\
& \operatorname{Tr}\left(\ell_{1}, 1, \ell_{2}, 4\right) \operatorname{Tr}\left(\ell_{1}, \ell_{2}, 2,3\right) A_{L}\left(\ell_{1}, 1, \ell_{2}, 4\right) A_{R}\left(-\ell_{1},-\ell_{2}, 2,3\right),
\end{aligned}
$$

and other three terms with $\left\{\ell_{1} \leftrightarrow \ell_{2}\right\}$. Thus we can get

$$
\begin{aligned}
\left.A_{3,1}(1,2,3 ; 4)\right|_{c u t}= & A_{L}\left(\ell_{1}, 1,2, \ell_{2}\right) A_{R}\left(-\ell_{1}, 4,-\ell_{2}, 3\right)+A_{L}\left(\ell_{1}, 3,1, \ell_{2}\right) A_{R}\left(-\ell_{1}, 4,-\ell_{2}, 2\right) \\
& +A_{L}\left(\ell_{1}, 1, \ell_{2}, 4\right) A_{R}\left(-\ell_{1},-\ell_{2}, 2,3\right)+\left\{\ell_{1} \leftrightarrow \ell_{2}\right\} .
\end{aligned}
$$

Then we can use KK relation (1.2) to put $\ell_{1}, \ell_{2}$ at the two ends

$$
A\left(\ell_{a}, i, \ell_{b}, j\right)=-A\left(\ell_{a}, i, j, \ell_{b}\right)-A\left(\ell_{a}, j, i, \ell_{b}\right)
$$

so (2.34) can be rewritten as

$$
\begin{aligned}
\left.A_{3,1}(1,2,3 ; 4)\right|_{\text {cut }}= & -A_{L}\left(\ell_{1}, 1,2, \ell_{2}\right) A_{R}\left(-\ell_{1},-\ell_{2}, 3,4\right)-A_{L}\left(\ell_{1}, 4,1, \ell_{2}\right) A_{R}\left(-\ell_{1},-\ell_{2}, 2,3\right) \\
& -A_{L}\left(\ell_{1}, 1,2, \ell_{2}\right) A_{R}\left(-\ell_{1},-\ell_{2}, 4,3\right)-A_{L}\left(\ell_{1}, 3,1, \ell_{2}\right) A_{R}\left(-\ell_{1},-\ell_{2}, 2,4\right) \\
& -A_{L}\left(\ell_{1}, 1,4, \ell_{2}\right) A_{R}\left(-\ell_{1},-\ell_{2}, 2,3\right)-A_{L}\left(\ell_{1}, 3,1, \ell_{2}\right) A_{R}\left(-\ell_{1},-\ell_{2}, 4,2\right) \\
& +\left\{\ell_{1} \leftrightarrow \ell_{2}\right\} .
\end{aligned}
$$

Comparing (2.36), (2.37), (2.38) with (2.33), it is clear that each line with its $\left\{\ell_{1} \leftrightarrow \ell_{2}\right\}$ terms can be identified as one primitive amplitude, so we get following identity between $A_{4,0}$ and $A_{3,1}$

$$
\begin{aligned}
A_{3,1}(1,2,3 ; 4) & =-A_{4,0}(1,2,3,4)-A_{4,0}(1,2,4,3)-A_{4,0}(1,4,2,3) \\
& =-\sum_{\sigma \in \text { cyclic }} A_{4,0}\left(\sigma_{1}, \sigma_{2}, \sigma_{3}, 4\right) .
\end{aligned}
$$

Similar argument can be applied to another double trace structure $A_{2,2}(1,2 ; 3,4)$. By working out the trace structures we can identify $\left.A_{2,2}(1,2 ; 3,4)\right|_{\text {cut }}$ as

$$
\begin{aligned}
\left.A_{2,2}(1,2 ; 3,4)\right|_{c u t}= & A_{L}\left(\ell_{1}, 1,2, \ell_{2}\right) A_{R}\left(-\ell_{1}, 3,4,-\ell_{2}\right)+A_{L}\left(\ell_{1}, 1,2, \ell_{2}\right) A_{R}\left(-\ell_{1}, 4,3,-\ell_{2}\right) \\
& +A_{L}\left(\ell_{1}, 1, \ell_{2}, 3\right) A_{R}\left(-\ell_{1}, 4,-\ell_{2}, 2\right)+A_{L}\left(\ell_{1}, 1, \ell_{2}, 4\right) A_{R}\left(-\ell_{1}, 3,-\ell_{2}, 2\right) \\
& +A_{L}\left(\ell_{1}, 2,1, \ell_{2}\right) A_{R}\left(-\ell_{1}, 3,4,-\ell_{2}\right)+A_{L}\left(\ell_{1}, 2,1, \ell_{2}\right) A_{R}\left(-\ell_{1}, 4,3,-\ell_{2}\right) \\
& +\left\{\ell_{1} \leftrightarrow \ell_{2}\right\} .
\end{aligned}
$$

Using the following KK relation

$$
A\left(\ell_{a}, \ell_{b}, i, j\right)=A\left(\ell_{a}, j, i, \ell_{b}\right), \quad A\left(\ell_{a}, i, \ell_{b}, j\right)=-A\left(\ell_{a}, i, j, \ell_{b}\right)-A\left(\ell_{a}, j, i, \ell_{b}\right),
$$


$\left.A_{2,2}(1,2 ; 3,4)\right|_{\text {cut }}$ can be written as

$$
\begin{aligned}
\left.A_{2,2}(1,2 ; 3,4)\right|_{c u t}= & A_{L}\left(\ell_{1}, 1,2, \ell_{2}\right) A_{R}\left(-\ell_{1},-\ell_{2}, 4,3\right)+A_{L}\left(\ell_{1}, 3,1, \ell_{2}\right) A_{R}\left(-\ell_{1},-\ell_{2}, 2,4\right) \\
& +A_{L}\left(\ell_{1}, 1,3, \ell_{2}\right) A_{R}\left(-\ell_{1},-\ell_{2}, 2,4\right)+A_{L}\left(\ell_{1}, 4,1, \ell_{2}\right) A_{R}\left(-\ell_{1},-\ell_{2}, 3,2\right) \\
& +A_{L}\left(\ell_{1}, 1,2, \ell_{2}\right) A_{R}\left(-\ell_{1},-\ell_{2}, 3,4\right)+A_{L}\left(\ell_{1}, 4,1, \ell_{2}\right) A_{R}\left(-\ell_{1},-\ell_{2}, 2,3\right) \\
& +A_{L}\left(\ell_{1}, 1,3, \ell_{2}\right) A_{R}\left(-\ell_{1},-\ell_{2}, 4,2\right)+A_{L}\left(\ell_{1}, 2,1, \ell_{2}\right) A_{R}\left(-\ell_{1},-\ell_{2}, 3,4\right) \\
& +A_{L}\left(\ell_{1}, 3,1, \ell_{2}\right) A_{R}\left(-\ell_{1},-\ell_{2}, 4,2\right)+A_{L}\left(\ell_{1}, 1,4, \ell_{2}\right) A_{R}\left(-\ell_{1},-\ell_{2}, 2,3\right) \\
& +A_{L}\left(\ell_{1}, 1,4, \ell_{2}\right) A_{R}\left(-\ell_{1},-\ell_{2}, 3,2\right)+A_{L}\left(\ell_{1}, 2,1, \ell_{2}\right) A_{R}\left(-\ell_{1},-\ell_{2}, 4,3\right) \\
& +\left\{\ell_{1} \leftrightarrow \ell_{2}\right\} .
\end{aligned}
$$

Each line with its $\left\{\ell_{1} \leftrightarrow \ell_{2}\right\}$ terms in above result corresponds to one of primitive amplitude $A_{4,0}$, and the whole result is nothing but

$$
\begin{aligned}
A_{2,2}(1,2 ; 3,4)= & A_{4,0}(1,2,3,4)+A_{4,0}(1,3,2,4)+A_{4,0}(1,3,4,2) \\
& +A_{4,0}(1,2,4,3)+A_{4,0}(1,4,2,3)+A_{4,0}(1,4,3,2) \\
= & \sum_{\sigma \in C O P\{1,2\} \cup\{3,4\}} A_{4,0}(\sigma) .
\end{aligned}
$$

Until now we have expressed the partial amplitudes of double trace structure as a linear combination of primitive amplitudes $A_{4,0}$, but among these primitive amplitudes, how many are really independent basis? Using the unitarity cut method, it is easy to see that for arbitrary $n$ we have (we will give a proof later)

$$
A_{n, 0}(1,2,3, \ldots, n-1, n)=(-)^{n} A_{n, 0}(n, n-1, \ldots, 2,1) .
$$

Thus for four-point primitive amplitudes, when accounting the cyclic symmetry and reflection identity (2.43), there are totally $S_{4} /\left(Z_{4} \times 2\right)=3$ independent primitive amplitudes. Is there any further relation among these three amplitudes as in the case of tree-level amplitudes? In order to answer this question, let us investigate cuts of these three amplitudes:

$$
\begin{aligned}
\left.A_{4,0}(1,2,3,4)\right|_{c u t}= & A_{L}\left(\ell_{1}, 1,2, \ell_{2}\right) A_{R}\left(-\ell_{2}, 3,4,-\ell_{1}\right)+A_{L}\left(\ell_{1}, 4,1, \ell_{2}\right) A_{R}\left(-\ell_{2}, 2,3,-\ell_{1}\right) \\
& +A_{L}\left(\ell_{1}, 2,1, \ell_{2}\right) A_{R}\left(-\ell_{2}, 4,3,-\ell_{1}\right)+A_{L}\left(\ell_{1}, 1,4, \ell_{2}\right) A_{R}\left(-\ell_{2}, 3,2,-\ell_{1}\right), \\
\left.A_{4,0}(1,3,2,4)\right|_{c u t}= & A_{L}\left(\ell_{1}, 1,3, \ell_{2}\right) A_{R}\left(-\ell_{2}, 2,4,-\ell_{1}\right)+A_{L}\left(\ell_{1}, 4,1, \ell_{2}\right) A_{R}\left(-\ell_{2}, 3,2,-\ell_{1}\right) \\
& +A_{L}\left(\ell_{1}, 3,1, \ell_{2}\right) A_{R}\left(-\ell_{2}, 4,2,-\ell_{1}\right)+A_{L}\left(\ell_{1}, 1,4, \ell_{2}\right) A_{R}\left(-\ell_{2}, 2,3,-\ell_{1}\right), \\
\left.A_{4,0}(1,3,4,2)\right|_{c u t}= & A_{L}\left(\ell_{1}, 1,3, \ell_{2}\right) A_{R}\left(-\ell_{2}, 4,2,-\ell_{1}\right)+A_{L}\left(\ell_{1}, 2,1, \ell_{2}\right) A_{R}\left(-\ell_{2}, 3,4,-\ell_{1}\right) \\
& +A_{L}\left(\ell_{1}, 3,1, \ell_{2}\right) A_{R}\left(-\ell_{2}, 2,4,-\ell_{1}\right)+A_{L}\left(\ell_{1}, 1,2, \ell_{2}\right) A_{R}\left(-\ell_{2}, 4,3,-\ell_{1}\right) .
\end{aligned}
$$

Let us focus on $s_{12}$ cut, which appears only in $A_{4,0}(1,2,3,4)$ and $A_{4,0}(1,3,4,2)$. The KK relation enables us to fix two legs, so let us choose the basis with fixing $\ell_{1}, \ell_{2}$, then $A_{L}$ and $A_{R}$ in above expression are 
already basis ${ }^{8}$. It is clear that terms in $A_{4,0}(1,2,3,4)$ and $A_{4,0}(1,3,4,2)$ are in different basis, which of course can not be related by algebraic relations. Thus the unitarity cut method tells us that there is no KK-like relation for four-point one-loop primitive amplitudes.

\subsubsection{Proof of general case}

After the example of four-point, we move to general $n$-point one-loop amplitude and try to prove (2.2). The proof will be given in following four steps. In the first step we will identify all cuts of different color trace structures and prove the color-ordering reversed identity (or reflection identity). In the second step, we will discuss example of $A_{n-1,1}$ to warm up. In the third step, we will present the proof of general case. In the last step, a technical detail will be explained.

The first step: We should identify all cut contributions of a given trace structure. For the partial amplitudes $A_{n, 0}$ (primitive amplitude), we have the following equation:

$$
\left.A_{n, 0}(1,2, \ldots, n)\right|_{\text {cut }}=\sum_{i=2}^{n-2} \sum_{P C P\{1,2, \ldots, n\}} A_{L}\left(\ell_{1}, 1,2, \ldots, i, \ell_{2}\right) A_{R}\left(-\ell_{2}, i+1, \ldots, n,-\ell_{1}\right)+\left\{\ell_{1} \leftrightarrow \ell_{2}\right\}
$$

where $P C P\{\sigma\}$ is the partially cyclic permutation of $(1,2, \ldots, n)$ such that particle 1 is always at the $A_{L}{ }^{9}$. For example, we have, for $i=3$,

$$
\begin{aligned}
\sum_{P C P\{1,2,3,4,5\}} A_{L}\left(\ell_{1}, 1,2,3, \ell_{2}\right) A_{R}\left(-\ell_{2}, 4,5,-\ell_{1}\right)= & A_{L}\left(\ell_{1}, 1,2,3, \ell_{2}\right) A_{R}\left(-\ell_{2}, 4,5,-\ell_{1}\right) \\
& +A_{L}\left(\ell_{1}, 5,1,2, \ell_{2}\right) A_{R}\left(-\ell_{2}, 3,4,-\ell_{1}\right) \\
& +A_{L}\left(\ell_{1}, 4,5,1, \ell_{2}\right) A_{R}\left(-\ell_{2}, 2,3,-\ell_{1}\right) .
\end{aligned}
$$

Using the explicit expression (2.44) we can show the reflection identity mentioned in (2.43). Using reflection identity for tree-level amplitudes $A_{L}, A_{R}$ we get

$$
\begin{aligned}
\left.A_{n, 0}(1,2, \ldots, n)\right|_{\text {cut }}= & \sum_{i=2}^{n-2} \sum_{P C P\{1,2, \ldots, n\}}(-)^{n+4} A_{L}\left(\ell_{2}, i, \ldots, 2,1, \ell_{1}\right) A_{R}\left(-\ell_{1}, n, \ldots, i+1,-\ell_{2}\right)+\left\{\ell_{1} \leftrightarrow \ell_{2}\right\} \\
= & (-)^{n} \sum_{i=2}^{n-2} \sum_{P C P\{1,2, \ldots, n\}} A_{L}\left(\ell_{1}, 1, n, \ldots, n-i+2, \ell_{2}\right) A_{R}\left(-\ell_{2}, n-i+1, \ldots, 2,-\ell_{1}\right) \\
& +\left\{\ell_{1} \leftrightarrow \ell_{2}\right\}
\end{aligned}
$$

where in the second line we have used the cyclic property under PCP. Summing up all terms in the second line gives $(-)^{n} A_{n, 0}(1, n, \ldots, 2)$, thus we have the reflection identity for primitive amplitude as given in (2.43).

\footnotetext{
${ }^{8}$ We will not consider the BCJ relation in this paper because the appearance of kinematic factors $s_{i j}$. We will remark this point in the conclusion.

${ }^{9}$ It is worth to notice that different $i$ will give different $P C P\{\sigma\}$.
} 
For partial amplitudes with double trace structure, for example, the $A_{c-1, n-c+1}(1,2, \ldots, c-1 ; c, \ldots, n)$, where $c \geq 2$, all double cuts are

$$
\left.A_{c-1, n-c+1}(1,2, \ldots, c-1 ; c, \ldots, n)\right|_{c u t}=\sum_{i \geq k}^{n-c+k+1} \sum_{k=1}^{c-1} \sum_{P C P\{1, \ldots, c-1\}} \sum_{C P\{c, \ldots, n\}} A_{L} A_{R}+\left\{\ell_{1} \leftrightarrow \ell_{2}\right\}
$$

where

$$
A_{L} A_{R}=A_{L}\left(\ell_{1}, 1, \ldots, k, \ell_{2}, n-i+k+1, \ldots, n\right) A_{R}\left(-\ell_{2}, k+1, \ldots, c-1,-\ell_{1}, c, \ldots, n-i+k\right)
$$

and $C P\{\alpha\}$ is the cyclic permutation over the set $\alpha$. The difference between $P C P$ and $C P$ is that in $P C P$ we require the particle 1 is always at the $A_{L}$ to avoid the double counting problem. Notice that $C P$ acts on the set $\{c, \ldots, n\}$, while $P C P$ acts on remaining set $\{1, \ldots, c-1\}$.

Similarly to $A_{n, 0}$, there is also a reflection identity. By accounting the $\left\{\ell_{1} \leftrightarrow \ell_{2}\right\}$ terms and using the reflection identity for tree-level amplitudes $A_{L}$ and $A_{R}$, we get

$$
\begin{aligned}
A_{L} A_{R}+\left\{\ell_{1} \leftrightarrow \ell_{2}\right\}= & (-)^{n+4} A_{L}\left(\ell_{1}, 1, c-1, \ldots, c-k+1, \ell_{2}, n, \ldots, n-i+k+1\right) \\
& \times A_{R}\left(-\ell_{2}, c-k, \ldots, 2,-\ell_{1}, n-i+k, \ldots, c\right)+\left\{\ell_{1} \leftrightarrow \ell_{2}\right\} \\
= & \left.(-)^{n} A_{c-1, n-c+1}(1, c-1, \ldots, 2 ; n-i+k, \ldots, c, n, \ldots, n-i+k+1)\right|_{\text {cut }} .
\end{aligned}
$$

Using the cyclic permutation invariant of each trace, we get the reflection identity

$$
A_{c-1, n-c+1}(1,2, \ldots, c-1 ; c, \ldots, n)=(-)^{n} A_{c-1, n-c+1}(c-1, c-2, \ldots, 1 ; n, n-1, \ldots, c) .
$$

The second step: As a warm up, we consider the relation between $A_{n-1,1}$ and $A_{n, 0}$. The cuts of $A_{n-1,1}$ are

$$
\begin{aligned}
\left.A_{n-1,1}(1,2, \ldots, n-1 ; n)\right|_{\text {cut }}= & \sum_{i=2}^{n-2} \sum_{P C P\{1,2, \ldots, n-1\}} A_{L}\left(\ell_{1}, 1,2, \ldots, i, \ell_{2}\right) A_{R}\left(-\ell_{2}, i+1, \ldots, n-1,-\ell_{1}, n\right) \\
& +\sum_{i=2}^{n-2} \sum_{P C P\{1,2, \ldots, n-1\}} A_{L}\left(\ell_{1}, 1,2, \ldots, i-1, \ell_{2}, n\right) A_{R}\left(-\ell_{2}, i, \ldots, n-1,-\ell_{1}\right) \\
& +\left\{\ell_{1} \leftrightarrow \ell_{2}\right\} .
\end{aligned}
$$


Using KK relation to put $\ell_{1}, \ell_{2}$ at two ends, we get

$$
\begin{aligned}
& \left.A_{n-1,1}(1,2, \ldots, n-1 ; n)\right|_{\text {cut }} \\
= & -\sum_{i=2}^{n-2} \sum_{P C P\{1,2, \ldots, n-1\}} \sum_{O P\{i+1, \ldots, n-1\} \cup\{n\}} A_{L}\left(\ell_{1}, 1,2, \ldots, i, \ell_{2}\right) A_{R}\left(-\ell_{2}, i+1, \ldots, n,-\ell_{1}\right) \\
& -\sum_{i=2}^{n-2} \sum_{P C P\{1,2, \ldots, n-1\}} \sum_{O P\{1, \ldots, i-1\} \cup\{n\}} A_{L}\left(\ell_{1}, 1, \ldots, i-1, n, \ell_{2}\right) A_{R}\left(-\ell_{2}, i, \ldots, n-1,-\ell_{1}\right)+\left\{\ell_{1} \leftrightarrow \ell_{2}\right\} \\
= & -\sum_{i=2}^{n-2} \sum_{P C P\{1,2, \ldots, n-1\}} \sum_{O P\{1, \ldots, n-1\} \cup\{n\}} A_{L}\left(\ell_{1}, 1, \ldots, i, \ell_{2}\right) A_{R}\left(-\ell_{2}, i+1, \ldots, n,-\ell_{1}\right)+\left\{\ell_{1} \leftrightarrow \ell_{2}\right\} .
\end{aligned}
$$

In order to express above terms as primitive amplitudes, we need to use following identity

$$
\begin{aligned}
& \sum_{P C P\{1,2, \ldots, n-1\}} \sum_{O P\{1, \ldots, n-1\} \cup\{n\}} A_{L}\left(\ell_{1}, 1, \ldots, i, \ell_{2}\right) A_{R}\left(-\ell_{2}, i+1, \ldots, n,-\ell_{1}\right) \\
= & \sum_{O P\{2,3, \ldots, n-1\} \cup\{n\}} \sum_{P C P\{1, \ldots, n\}} A_{L}\left(\ell_{1}, 1, \ldots, i, \ell_{2}\right) A_{R}\left(-\ell_{2}, i+1, \ldots, n,-\ell_{1}\right) .
\end{aligned}
$$

So the final result would be

$$
\begin{aligned}
& \left.A_{n-1,1}(1,2, \ldots, n-1 ; n)\right|_{\text {cut }} \\
= & -\sum_{O P\{2,3, \ldots, n-1\} \cup\{n\}} \sum_{i=2}^{n-2} \sum_{P C P\{1, \ldots, n\}} A_{L}\left(\ell_{1}, 1, \ldots, i, \ell_{2}\right) A_{R}\left(-\ell_{2}, i+1, \ldots, n,-\ell_{1}\right) \\
= & -\left.\sum_{\alpha \in O P\{2,3, \ldots, n-1\} \cup\{n\}} A_{n, 0}(1, \alpha)\right|_{\text {cut }}=-\left.\sum_{\beta \in \text { cyclic }} A_{n, 0}\left(\beta_{1}, \beta_{2}, \ldots, \beta_{n-1}, n\right)\right|_{\text {cut }},
\end{aligned}
$$

which is a special case of general formula (2.2).

The third step: Now we consider the general case (2.45). After using KK relation to $A_{L} A_{R}$, a typical term of (2.45) will become

$$
\begin{aligned}
& (-1)^{n-c+1} \sum_{\sigma \in O P\{1, \ldots, k\} \cup\{n, \ldots, n-i+k+1\}} A_{L}\left(\ell_{1}, \sigma(1, \ldots, k, n, \ldots, n-i+k+1), \ell_{2}\right) \\
& \times \sum_{\tilde{\sigma} \in O P\{k+1, \ldots, c-1\} \cup\{n-i+k, \ldots, c\}} A_{R}\left(-\ell_{2}, \widetilde{\sigma}(k+1, \ldots, c-1, n-i+k, \ldots, c),-\ell_{1}\right),
\end{aligned}
$$

where the ordering $\{c, c+1, \ldots, n\}$ has been reversed by the KK relation. Other terms with given $k, i$ are obtained by cyclic permutation of $k$-elements from set $\{1,2, \ldots, c-1\}$ and $(i-k)$-elements from the set $\{n, \ldots, c\}$. Finally we need to sum up all allowed $k, i$. Regrouping them together, we can rewrite 
$\left.A_{c-1, n-c+1}\right|_{c u t}$ as

$$
\left.A_{c-1, n-c+1}(1, \ldots, c-1 ; c, \ldots, n)\right|_{c u t}=(-1)^{n-c+1} \sum_{i=2}^{n-2} \sum_{P C P\{1, \ldots, c-1\}} \sum_{C P\{c, \ldots, n\}} \sum_{P O P\{1, \ldots, c-1\} \cup\{n, \ldots, c\}} A_{L} A_{R},
$$

where

$$
A_{L} A_{R}=A_{L}\left(\ell_{1}, 1, \ldots, i, \ell_{2}\right) A_{R}\left(-\ell_{2}, i+1, \ldots, n,-\ell_{1}\right)
$$

and $P O P\{\alpha\} \cup\{\beta\}$ (Partially Ordered Permutation) means ordered permutations between sets $\{\alpha\}$ and $\{\beta\}$ while keeping 1 in $A_{L}$. Using identity

$$
\begin{aligned}
& \sum_{P C P\{1, \ldots, c-1\}} \sum_{C P\{c, \ldots, n\}} \sum_{P O P\{1, \ldots, c-1\} \cup\{n, \ldots, c\}} A_{L}\left(\ell_{1}, 1, \ldots, i, \ell_{2}\right) A_{R}\left(-\ell_{2}, i+1, \ldots, n,-\ell_{1}\right) \\
= & \sum_{C P\{c, \ldots, n\}} \sum_{O P\{2, \ldots, c-1\} \cup\{n, \ldots, c\}} A_{P C P\{1, \ldots, n\}}\left(\ell_{1}, 1, \ldots, i, \ell_{2}\right) A_{R}\left(-\ell_{2}, i+1, \ldots, n,-\ell_{1}\right),
\end{aligned}
$$

as well as (2.44), $\left.A_{c-1, n-c+1}\right|_{c u t}$ can be simplified as

$$
\left.A_{c-1, n-c+1}(1,2, \ldots, c-1 ; c, \ldots, n)\right|_{c u t}=\left.(-1)^{n-c+1} \sum_{C P\{c, \ldots, n\} O P\{2, \ldots, c-1\} \cup\{n, \ldots, c\}} A_{n, 0}(1,2, \ldots, n)\right|_{c u t} .
$$

The two summations which are over all permutations between set $\{1,2, \ldots, c-1\}$ and $\{n, \ldots, c\}$ with 1 fixed at the first position, preserve the cyclic ordering of set $\{n, \ldots, c\}$. They are nothing but familiar

$$
C O P\{1,2, \ldots, c-1\} \cup\{n, \ldots, c\}
$$

thus we finally prove

$$
A_{c-1, n-c+1}(1,2, \ldots, c-1 ; c, \ldots, n)=(-1)^{n-c+1} \sum_{C O P\{1,2, \ldots, c-1\} \cup\{n, \ldots, c\}} A_{n, 0}(1,2, \ldots, n)
$$

Using the reflection identity (2.46) we can obtain another form

$$
A_{c-1, n-c+1}(1,2, \ldots, c-1 ; c, \ldots, n)=(-1)^{c-1} \sum_{C O P\{c-1, \ldots, 1\} \cup\{c, c+1, \ldots, n\}} A_{n, 0}(1,2, \ldots, n)
$$

The last step: The remaining thing we should clarify is the identities (2.48) and (2.52). Since (2.48) is a special case of (2.52) when $c=n$, we just need to prove the identity (2.52). To do so, we will consider terms with leg 1 in the any given $k$-th position of ordering in $A_{L} A_{R}$. Since $k$ is chosen arbitrarily, if the 
terms at both sides match up, then the identity is true. Let us consider the summation of the first line in (2.52),

$$
\sum_{P C P\{1, \ldots, c-1\}} \sum_{C P\{c, \ldots, n\} P O P\{1, \ldots, c-1\} \cup\{n, \ldots, c\}} \sum_{L}\left(\ell_{1}, 1, \ldots, i, \ell_{2}\right) A_{R}\left(-\ell_{2}, i+1, \ldots, n,-\ell_{1}\right) .
$$

The ordering of first summation and second summation does not matter since they act on different sets. In order to hold leg 1 in the $k$-th position in $A_{L}$, we should first take $P O P$ action and then $P C P$ action. The final result where leg 1 is at the $k$-th position is

$$
\{1,2, \ldots, n\} \rightarrow\left\{O P\left\{\sigma_{k-1-m}\right\} \cup\left\{\sigma_{m}\right\}, 1, O P\left\{\sigma_{c+m-k-1}\right\} \cup\left\{\sigma_{n-c-m+1}\right\}\right\}
$$

where

$$
\left\{\sigma_{c+m-k-1}, \sigma_{k-1-m}\right\}=\{2, \ldots, c-1\}, \quad\left\{\sigma_{m}, \sigma_{n-c-m+1}\right\}=\{n, \ldots, c\} .
$$

The subscript of set $\sigma$ stands for the number of elements in $\sigma$, and $m$ takes the value that all four $\sigma$ sets are meaningful.

Then let us consider the summation of the second line in (2.52),

$$
\sum_{C P\{c, \ldots, n\}} \sum_{O P\{2, \ldots, c-1\} \cup\{n, \ldots, c\}} \sum_{P C P\{1, \ldots, n\}} A_{L}\left(\ell_{1}, 1, \ldots, i, \ell_{2}\right) A_{R}\left(-\ell_{2}, i+1, \ldots, n,-\ell_{1}\right) .
$$

In order to hold leg 1 at $k$-th position, we should simply take the following replacement using $P C P$,

$$
\{1,2, \ldots, n\} \rightarrow\{n-k+2, \ldots, n, 1,2, \ldots, n-k+1\}
$$

Since actions under $P O P$ and $C P$ will not change the position of leg 1 , we could then take the following replacements under $O P$

$$
\{1,2, \ldots, n\} \rightarrow\{1, O P\{2, \ldots, c-1\} \cup\{n, \ldots, c\}\}
$$

which means that $\{2,3, \ldots, n-k+1\}$ should be replaced by the front $(n-k)$ elements of $\{O P\{2, \ldots, c-1\} \cup$ $\{n, \ldots, c\}\}$, and $\{n-k+2, \ldots, n\}$ should be replaced by the remaining $(k-1)$ elements of $\{O P\{2, \ldots, c-$ $1\} \cup\{n, \ldots, c\}\}$. By setting

$$
\left\{\sigma_{c+m-k-1}^{\prime}, \sigma_{k-1-m}^{\prime}\right\}=\{2, \ldots, c-1\}, \quad\left\{\sigma_{n-c-m+1}^{\prime}, \sigma_{m}^{\prime}\right\}=\{n, \ldots, c\},
$$

the above replacements can be compactly written as

$$
\begin{aligned}
& \{2, \ldots, n-k+1\} \rightarrow\left\{O P\left\{\sigma_{c+m-k-1}^{\prime}\right\} \cup\left\{\sigma_{n-c-m+1}^{\prime}\right\}\right\} \\
& \{n-k+2, \ldots, n\} \rightarrow\left\{O P\left\{\sigma_{k-1-m}^{\prime}\right\} \cup\left\{\sigma_{m}^{\prime}\right\}\right\} .
\end{aligned}
$$

The final result of actions (2.60) and 2.63) is

$$
\{1,2, \ldots, n\} \rightarrow\left\{O P\left\{\sigma_{k-1-m}^{\prime}\right\} \cup\left\{\sigma_{m}^{\prime}\right\}, 1, O P\left\{\sigma_{c+m-k-1}^{\prime}\right\} \cup\left\{\sigma_{n-c-m+1}^{\prime}\right\}\right\}
$$


Until now (2.64) is not equal to (2.57), since we have $\left\{\sigma_{m}, \sigma_{n-c-m+1}\right\}=\{n, \ldots, c\}$ in (2.58) while $\left\{\sigma_{n-c-m+1}^{\prime}, \sigma_{m}^{\prime}\right\}=\{n, \ldots, c\}$ in (2.62). Thus the elements in $\sigma_{m}$ and $\sigma_{m}^{\prime}$ are different, and so are $\sigma_{n-c-m+1}$ and $\sigma_{n-c-m+1}^{\prime}$. But when considering sum of cyclic permutations $\sum_{C P\{c, \ldots, n\}}$ at both sides, we can rewrite $\left\{\sigma_{n-c-m+1}^{\prime}, \sigma_{m}^{\prime}\right\}$ as

$$
\left\{\sigma_{n-c-m+1}^{\prime}, \sigma_{m}^{\prime}\right\}=\{n-m+2, \ldots, c+1, c ; n, n-1, \ldots, n-m+1\},
$$

then we have $\sigma_{m}^{\prime}=\sigma_{m}=\{n, n-1, \ldots, n-m+1\}$, thus proving (2.52).

\section{Partial amplitudes of two-loop amplitude}

After one-loop calculation, we want to generalize our method to higher loop. In this section, we will focus on two-loop case. The color decomposition for two-loop amplitude in $U(N)$ gauge theory can be schematically written as

$$
\begin{aligned}
\mathcal{A}_{n}^{2-\text { loop }}= & \sum_{\sigma \in S_{n} / Z_{n}} N_{c}^{2} \operatorname{Tr}\left(\sigma_{1}, \ldots, \sigma_{n}\right)\left(A_{n}^{L C}\left(\sigma_{1}, \ldots, \sigma_{n}\right)+\frac{1}{N_{c}^{2}} A_{n}^{S C}\left(\sigma_{1}, \ldots, \sigma_{n}\right)\right) \\
& +\sum_{m=1}^{\lfloor n / 2\rfloor} \sum_{\sigma \in S_{n} / S_{n-m, m}} N_{c} \operatorname{Tr}\left(\sigma_{1}, \ldots, \sigma_{m}\right) \operatorname{Tr}\left(\sigma_{m+1}, \ldots, \sigma_{n}\right) A_{n-m, m}\left(\sigma_{1}, \ldots, \sigma_{m} ; \sigma_{m+1}, \ldots, \sigma_{n}\right) \\
& +\sum_{a=1}^{\lfloor n / 3\rfloor} \sum_{(b-a)=a}^{\lfloor(n-a) / 2\rfloor} \sum_{\sigma \in S_{n} / S_{a, b-a, n-b}} \operatorname{Tr}(\alpha) \operatorname{Tr}(\beta) \operatorname{Tr}(\gamma) A_{a, b-a, n-b}(\alpha ; \beta ; \gamma)
\end{aligned}
$$

where $\alpha=\left\{\sigma_{1}, \ldots, \sigma_{a}\right\}, \beta=\left\{\sigma_{a+1}, \ldots, \sigma_{b}\right\}$ and $\gamma=\left\{\sigma_{b+1}, \ldots, \sigma_{n}\right\} . S_{n-m, n}$ and $S_{n-b, b-a, a}$ are corresponding groups that leaving the double trace and triple trace invariant. The subscripts of partial amplitudes denote the number of generators in traces. There are two kinds of single trace structure: the ones with power $N_{c}^{2}$ are leading-color single trace amplitudes and the others are subleading-color single trace amplitudes, which come from non-planar Feynman diagrams. The partial amplitudes are gauge invariant and may be calculated separately.

For two-loop case, there are not many results on relations between partial amplitudes, due to the appearance of triple trace structure as well as the subleading-color single trace structure, which make the discussions more complicated. We would like to know, for example, if there are relations like (2.2), so all other partial amplitudes can be expressed by leading-color single trace partial amplitudes $A_{n, 0,0}$. If we can not achieve this goal, then how far we can go, i.e., what is the minimum basis of the partial amplitudes we need to completely determine the whole two-loop amplitudes. To answer these questions, we would rely on both $U(1)$-decoupling method and unitarity cut method.

The generalization of $U(1)$-decoupling method to two-loop is straightforward, and the only difference from one-loop case is the appearance of triple trace structure and subleading-color single trace structure, which will lead to more decoupling equations. The solving of all these equations is also more complicated. 
To generalize unitarity cut method to two-loop amplitude, we need to introduce the triple cut. Then the whole two-loop amplitude becomes (to prevent double-counting, we can fix leg 1 in $A_{L}$ )

$$
\left.\mathcal{A}_{n}^{2-\text { loop }}\right|_{\text {cut }}=\sum_{\text {states }} \sum_{\text {of } \ell_{1}, \ell_{2}, \ell_{3}} \sum_{L, R} \mathcal{A}_{L}^{\text {full tree }}\left(\ell_{1}, \ell_{2}, \ell_{3}, \sigma_{L}\right) \mathcal{A}_{R}^{\text {full tree }}\left(-\ell_{1},-\ell_{2},-\ell_{3}, \sigma_{R}\right),
$$

where the summation is over all allowed triple cuts. Like one-loop case, there are also a few technical points we need to point out. First for gauge theory, we assume that there is no contribution by reduction process with only two inner propagators ( so there is no triple cut available). It is the generalization of the fact that there is no tadpole contribution at one-loop for gauge theory. Secondly, we assume that there is basis for two-loop amplitudes without the topology that two one-loop diagrams are attached to each other at a vertex (such as the "bow-tie" diagram given in [46] or the "kissing box" diagrams given in [47]). Since there is still no fully understanding of basis of two loop amplitudes and how we can treat one basis to another basis ${ }^{10}$, we can not show the assumption to be true, thus our results in this section should be taken with caution up to this uncertainty. For the two loop MHV-amplitudes of $\mathcal{N}=4$ theory, Drummond and Henn 48] have shown how to tread the kissing box diagram to diagrams satisfying our assumption. Thirdly we require there are at least two external gluons at $A_{L}$ and $A_{R}$, which is also reasonable for massless theory ${ }^{11}$. Fourthly we assume our triple cut discussion is true for general $(4-2 \epsilon)$-dimension. Otherwise our conclusion is true only for the $\mathcal{N}=4$ theory. Our following discussions will base on above four technical assumptions.

To see color structures of partial amplitudes coming from the triple cut method, we do similar calculations as in (2.26)

$$
\begin{aligned}
& \sum_{\ell_{i}}\left(\operatorname{Tr}\left(\ell_{1}, \alpha_{L}, \ell_{2}, \beta_{L}, \ell_{3}, \gamma_{L}\right)+\operatorname{Tr}\left(\ell_{1}, \widetilde{\alpha}_{L}, \ell_{3}, \widetilde{\beta}_{L}, \ell_{2}, \widetilde{\gamma}_{L}\right)\right) \\
& \times\left(\operatorname{Tr}\left(\ell_{1}, \alpha_{R}, \ell_{2}, \beta_{R}, \ell_{3}, \gamma_{R}\right)+\operatorname{Tr}\left(\ell_{1}, \widetilde{\alpha}_{R}, \ell_{3}, \widetilde{\beta}_{R}, \ell_{2}, \widetilde{\gamma}_{R}\right)\right) \\
= & \operatorname{Tr}\left(\gamma_{L}, \alpha_{R}, \beta_{L}, \gamma_{R}, \alpha_{L}, \beta_{R}\right)+\operatorname{Tr}\left(\widetilde{\beta}_{L}, \widetilde{\gamma}_{T}, \widetilde{\alpha}_{L}, \widetilde{\beta}_{R}, \widetilde{\gamma}_{L}, \widetilde{\alpha}_{R}\right) \\
+ & \operatorname{Tr}\left(\gamma_{L}, \widetilde{\alpha}_{R}\right) \operatorname{Tr}\left(\widetilde{\beta}_{R}, \beta_{L}\right) \operatorname{Tr}\left(\widetilde{\gamma}_{R}, \alpha_{L}\right)+\operatorname{Tr}\left(\gamma_{R}, \widetilde{\alpha}_{L}\right) \operatorname{Tr}\left(\widetilde{\beta}_{L}, \beta_{R}\right) \operatorname{Tr}\left(\widetilde{\gamma}_{L}, \alpha_{R}\right),
\end{aligned}
$$

which reproduces the familiar color structures given in (3.1). The first two terms come from

$$
\operatorname{Tr}\left(\ell_{1}, \ldots, \ell_{2}, \ldots, \ell_{3}, \ldots\right) \operatorname{Tr}\left(\ell_{1}, \ldots, \ell_{2}, \ldots, \ell_{3}, \ldots\right), \quad \operatorname{Tr}\left(\ell_{1}, \ldots, \ell_{3}, \ldots, \ell_{2}, \ldots\right) \operatorname{Tr}\left(\ell_{1}, \ldots, \ell_{3}, \ldots, \ell_{2}, \ldots\right)
$$

which contribute to subleading-color single trace structure, while the other two terms come from

$$
\operatorname{Tr}\left(\ell_{1}, \ldots, \ell_{2}, \ldots, \ell_{3}, \ldots\right) \operatorname{Tr}\left(\ell_{1}, \ldots, \ell_{3}, \ldots, \ell_{2}, \ldots\right), \quad \operatorname{Tr}\left(\ell_{1}, \ldots, \ell_{3}, \ldots, \ell_{2}, \ldots\right) \operatorname{Tr}\left(\ell_{1}, \ldots, \ell_{2}, \ldots, \ell_{3}, \ldots\right)
$$

\footnotetext{
${ }^{10}$ There is a very nice paper [49] discussing the basis of planar two-loop integrals.

${ }^{11}$ One argument for this is as follows. For one-loop case, the integration $\int d^{D} \ell \frac{1}{\ell^{2}(\ell-k)^{2}}$ tell us that its form should be $\left(k^{2}\right)^{\frac{D-4}{2}}$ by dimension analysis. Even with the tensor structure in numerator, we will still see the appearing of factor $k^{2}$ with proper power. This is one reason why massless bubble gives zero contribution. For two loop with massless external momenta such as $\frac{f\left(\ell_{i}\right)}{\ell_{1}^{2} \ell_{2}^{2}\left(\ell_{1}+\ell_{2}+k\right)^{2}}$, similar consideration implies result $\left(k^{2}\right)^{\frac{2 D-6}{2}}$ for numerator $f(\ell)=1$, or $k_{\mu_{1}} k_{\mu_{2}} \ldots k_{\mu_{i}}\left(k^{2}\right)^{\frac{2 D-6}{2}+\frac{n-i}{2}}$ when $f(\ell)$ is tensor structure. Thus dimensional regularization implies the final result should be zero.
} 
which contribute to leading-color single, double and triple trace structures, depending on how many empty sets in these two terms are. It is very important to notice that from above discussions the pattern of contributions to subleading-color single trace is different from those of other trace structures, so these two types will not mix with each other in a simple way.

One simple result coming from the triple cut method is the reflection identity for any type of partial amplitudes

$$
A_{a, b, n-a-b}(\alpha ; \beta ; \gamma)=(-)^{n} A_{a, b, n-a-b}\left(\alpha^{T} ; \beta^{T} ; \gamma^{T}\right)
$$

where $T$ means the reversing of ordering.

Having the experience of one-loop case, in this section, our discussion will be more briefly. On the other hand, because the difficulty of the problem, we have only some preliminary results and more works need to be done in future.

\subsection{Understanding four-point amplitude from $U(1)$-decoupling method}

Again we will start with the simplest example, i.e., the four-point two loop amplitudes. We will use the $U(1)$-decoupling method in this subsection and triple cut method in next subsection. It is worth to remember that our discussion of $U(1)$-decoupling equation is not new, and results in this subsection can be found, for example, in [50] (see also [51]). The purpose of this subsection is to set up identities, so we can test our generalized unitarity cut method in next subsection.

The color decomposition of four-point amplitude is 43]

$$
\begin{aligned}
\mathcal{A}_{4}^{2-\text { loop }}= & \sum_{\sigma \in S_{4} / Z_{4}} N_{c}^{2}\left(\operatorname{Tr}\left(\sigma_{1}, \sigma_{2}, \sigma_{3}, \sigma_{4}\right) A_{4}^{L C}\left(\sigma_{1}, \sigma_{2}, \sigma_{3}, \sigma_{4}\right)+\frac{1}{N_{c}^{2}} \operatorname{Tr}\left(\sigma_{1}, \sigma_{2}, \sigma_{3}, \sigma_{4}\right) A_{4}^{S C}\left(\sigma_{1}, \sigma_{2}, \sigma_{3}, \sigma_{4}\right)\right) \\
& +\sum_{\sigma \in S_{4} / Z_{3}} N_{c} \operatorname{Tr}\left(\sigma_{1}\right) \operatorname{Tr}\left(\sigma_{2}, \sigma_{3}, \sigma_{4}\right) A_{1,3}\left(\sigma_{1} ; \sigma_{2}, \sigma_{3}, \sigma_{4}\right)+\sum_{\sigma \in S_{4} / Z_{2}^{3}} N_{c} \operatorname{Tr}\left(\sigma_{1}, \sigma_{2}\right) \operatorname{Tr}\left(\sigma_{3}, \sigma_{4}\right) A_{2,2}\left(\sigma_{1}, \sigma_{2} ; \sigma_{3}, \sigma_{4}\right) \\
& +\sum_{\sigma \in S_{4} / Z_{2}^{2}} \operatorname{Tr}\left(\sigma_{1}, \sigma_{2}\right) \operatorname{Tr}\left(\sigma_{3}\right) \operatorname{Tr}\left(\sigma_{4}\right) A_{2,1,1}\left(\sigma_{1}, \sigma_{2} ; \sigma_{3} ; \sigma_{4}\right)
\end{aligned}
$$

where the summation for each color trace structure is over all distinguished permutations, i.e., we should mod out permutations making the color trace structure invariant.

There are five kinds of trace structures: the subleading-color single trace, the leading-color single trace, the double trace $(3 \mid 1)$ and $(2 \mid 2)$, and finally the triple trace $(1|1| 2)$. By setting generators to be $U(1)$, subleading-color single trace can never be mixed with other color structures, so they have relations only among themselves ${ }^{12}$. For the remaining color structures, by setting one generator to be $U(1)$, they reduce to

$$
(4) \rightarrow(3) ; \quad(3 \mid 1) \rightarrow(3) \text { or }(1 \mid 2) ; \quad(2 \mid 2) \rightarrow(1 \mid 2) ; \quad(1|1| 2) \rightarrow(1 \mid 2) \text { or }(1|1| 1) .
$$

\footnotetext{
${ }^{12}$ Another way to see it is that we can take $N_{c}$ as free parameter, so a function is zero when and only when all coefficients of different $N_{c}$-power to be zero.
} 
Thus the reduced trace structure (3) gives a relation between $A_{4}^{L C}$ and $A_{1,3}$. The reduced (1|2) structure gives a relation among $A_{1,3}, A_{2,2}$ and $A_{2,1,1}$ and finally the reduced (1|1|1) structure gives a relation of $A_{2,1,1}$.

More explicitly, by setting $T^{4}$ as $U(1)$, for the partial amplitudes of subleading-color single trace, we get

$$
0=A_{4}^{S C}(4,1,2,3)+A_{4}^{S C}(4,3,1,2)+A_{4}^{S C}(4,2,3,1)
$$

It is worth to notice that it is exact the same form as tree-level $U(1)$-decoupling equation. Then it is interesting to ask if there is the same KK relation for subleading-color single partial amplitudes? This question has no hint from $U(1)$-decoupling method, but can be investigated by triple cut method in late subsection.

Let us continue to other $U(1)$-decoupling relation. From the reduced $N_{c}^{2} \operatorname{Tr}(1,2,3)$ structure we can read out

$$
0=A_{1,3}(4 ; 1,2,3)+\sum_{\text {cyclic }(123)} A_{4}^{L C}(4,1,2,3)
$$

so we can solve (other $A_{1,3}$ can be obtained simply by relabeling)

$$
A_{1,3}(4 ; 1,2,3)=-A_{4}^{L C}(4,1,2,3)-A_{4}^{L C}(4,3,1,2)-A_{4}^{L C}(4,2,3,1) .
$$

From the reduced $N_{c} \operatorname{Tr}(1) \operatorname{Tr}(2,3)$ structure we have

$$
0=A_{1,3}(1 ; 4,2,3)+A_{1,3}(1 ; 4,3,2)+A_{2,2}(4,1 ; 2,3)+A_{2,1,1}(2,3 ; 1 ; 4),
$$

and finally from the reduced $\operatorname{Tr}(1) \operatorname{Tr}(2) \operatorname{Tr}(3)$ structure, we have

$$
0=A_{2,1,1}(4,1 ; 2 ; 3)+A_{1,2,1}(1 ; 4,2 ; 3)+A_{1,1,2}(1 ; 2 ; 4,3) .
$$

Other independent relations will be obtained by relabeling of indices.

Having these equations, we would like to ask if they are enough to solve all the $A_{1,1,2}$ and $A_{2,2}$ in terms of $A_{4}^{L C}$. Let us check this by solving with (3.11) firstly. There are $S_{4} / Z_{2} Z_{2}=6$ of $A_{1,1,2}$ and four equations, which can be written as

$\left.0\right|_{T_{4}=1}=X_{3}+X_{5}+X_{6},\left.\quad 0\right|_{T_{2}=1}=X_{1}+X_{4}+X_{5},\left.\quad 0\right|_{T_{3}=1}=X_{2}+X_{4}+X_{6},\left.\quad 0\right|_{T_{1}=1}=X_{1}+X_{2}+X_{3}$, with

$$
\begin{aligned}
& X_{1}=A_{2,1,1}(1,2 ; 3 ; 4), \quad X_{2}=A_{2,1,1}(1,3 ; 2 ; 4), \quad X_{3}=A_{2,1,1}(1,4 ; 2 ; 3) \\
& X_{4}=A_{2,1,1}(2,3 ; 1 ; 4), \quad X_{5}=A_{2,1,1}(2,4 ; 1 ; 3), \quad X_{6}=A_{2,1,1}(3,4 ; 1 ; 2) .
\end{aligned}
$$

From these equations we can solve

$$
X_{3}=-X_{1}-X_{2}, \quad X_{4}=-X_{1}-X_{2}, \quad X_{5}=X_{2}, \quad X_{6}=X_{1},
$$


where we have taken $X_{1}$ and $X_{2}$ as basis. Putting them into (3.10) we find solution for following three $A_{2,2}$ :

$$
Y_{1}=A_{2,2}(1,2 ; 3,4), \quad Y_{2}=A_{2,2}(1,3 ; 2,4), \quad Y_{3}=A_{2,2}(1,4 ; 2,3)
$$

as

$$
\begin{aligned}
& Y_{1}=-X_{1}+\sum_{\sigma \in C O P\{1,2\} \cup\{3,4\}} A_{4}^{L C}(\sigma), \quad Y_{2}=-X_{2}+\sum_{\sigma \in C O P\{1,3\} \cup\{2,4\}} A_{4}^{L C}(\sigma), \\
& Y_{3}=X_{1}+X_{2}+\sum_{\sigma \in C O P\{1,4\} \cup\{2,3\}} A_{4}^{L C}(\sigma),
\end{aligned}
$$

where the difference between one loop $A_{2,2}$ and two loop $A_{2,2}$ is the appearance of $A_{1,1,2}$ in (3.14).

In summary, from solving $U(1)$-decoupling equations, we see that partial amplitudes of subleadingcolor trace structure are themselves a special category, which has same $U(1)$-decoupling relation as the one for tree-level amplitudes. The remaining partial amplitudes can be expressed as linear combination of all three independent partial amplitudes of leading-color single trace structure, plus two partial amplitudes of double (or triple) trace structure.

\subsection{Further understanding of four-point amplitude from unitarity cut method}

All relations coming from $U(1)$-decoupling method in previous subsection can be directly verified by unitarity cut method. However, from one-loop example, we are warned that there are non-trivial relations that can not be solved directly from $U(1)$-decoupling relation. Thus we would like to ask are there any more relations that are not revealed in $U(1)$-decoupling relation? More specifically, we want to ask: (1) If we can express all partial amplitudes of double or triple trace as a linear combination of partial amplitudes of leading-color single trace? (2) If not, then we would like to ask if the basis given in previous subsection, which includes leading-color single trace and other two partial amplitudes (it could be two double (or triple) trace amplitudes), are independent to each other.

In this subsection, we will discuss these problems using unitarity cut method. Before going on, let us work out the cut structures of partial amplitudes. By a straightforward calculation, we can express the cuts of the partial amplitude of leading-color single trace as

$$
\begin{aligned}
\left.A_{4}^{L C}(1,2,3,4)\right|_{\text {cut }}= & A_{L}\left(\ell_{1}, 1,2, \ell_{2}, \ell_{3}\right) A_{R}\left(-\ell_{1},-\ell_{3},-\ell_{2}, 3,4\right)+A_{L}\left(\ell_{1}, 4,1, \ell_{2}, \ell_{3}\right) A_{R}\left(-\ell_{1},-\ell_{3},-\ell_{2}, 2,3\right) \\
& +P\left\{\ell_{1}, \ell_{2}, \ell_{3}\right\}
\end{aligned}
$$

where $P\left\{\ell_{1}, \ell_{2}, \ell_{3}\right\}$ means we should plus all the written terms with all the other permutations of $\left\{\ell_{1}, \ell_{2}, \ell_{3}\right\}$. 
Similarly for $A_{3,1}(1,2,3 ; 4)$ we have

$$
\begin{aligned}
\left.A_{3,1}(1,2,3 ; 4)\right|_{\text {cut }}= & A_{L}\left(\ell_{1}, 1,2, \ell_{2}, \ell_{3}\right) A_{R}\left(-\ell_{1},-\ell_{3}, 4,-\ell_{2}, 3\right)+A_{L}\left(\ell_{1}, 1,2, \ell_{2}, \ell_{3}\right) A_{R}\left(-\ell_{1}, 4,-\ell_{3},-\ell_{2}, 3\right) \\
& +A_{L}\left(\ell_{1}, 3,1, \ell_{2}, \ell_{3}\right) A_{R}\left(-\ell_{1},-\ell_{3}, 4,-\ell_{2}, 2\right)+A_{L}\left(\ell_{1}, 3,1, \ell_{2}, \ell_{3}\right) A_{R}\left(-\ell_{1}, 4,-\ell_{3},-\ell_{2}, 2\right) \\
& +A_{L}\left(\ell_{1}, 1, \ell_{2}, 4, \ell_{3}\right) A_{R}\left(-\ell_{1},-\ell_{3},-\ell_{2}, 2,3\right)+A_{L}\left(\ell_{1}, 1, \ell_{2}, \ell_{3}, 4\right) A_{R}\left(-\ell_{1},-\ell_{3},-\ell_{2}, 2,3\right) \\
& +P\left\{\ell_{1}, \ell_{2}, \ell_{3}\right\}
\end{aligned}
$$

and for $A_{2,2}(1,2 ; 3,4)$,

$$
\begin{aligned}
\left.A_{2,2}(1,2 ; 3,4)\right|_{\text {cut }}= & A_{L}\left(\ell_{1}, \alpha_{1}, \alpha_{2}, \ell_{2}, \ell_{3}\right) A_{R}\left(-\ell_{1},-\ell_{3}, \beta_{3}, \beta_{4},-\ell_{2}\right)+A_{L}\left(\ell_{1}, \alpha_{1}, \alpha_{2}, \ell_{2}, \ell_{3}\right) A_{R}\left(-\ell_{1}, \beta_{3}, \beta_{4},-\ell_{3},-\ell_{2}\right) \\
& A_{L}\left(\ell_{1}, 1, \ell_{2}, 3, \ell_{3}\right) A_{R}\left(-\ell_{1},-\ell_{3}, 4,-\ell_{2}, 2\right)+A_{L}\left(\ell_{1}, 1, \ell_{2}, \ell_{3}, 3\right) A_{R}\left(-\ell_{1}, 4,-\ell_{3},-\ell_{2}, 2\right) \\
& A_{L}\left(\ell_{1}, 1, \ell_{2}, 4, \ell_{3}\right) A_{R}\left(-\ell_{1},-\ell_{3}, 3,-\ell_{2}, 2\right)+A_{L}\left(\ell_{1}, 1, \ell_{2}, \ell_{3}, 4\right) A_{R}\left(-\ell_{1}, 3,-\ell_{3},-\ell_{2}, 2\right) \\
& +P\left\{\ell_{1}, \ell_{2}, \ell_{3}\right\}
\end{aligned}
$$

where $\alpha, \beta \in Z_{2}$. Finally the expression for the cuts of partial amplitude of triple trace structure $A_{2,1,1}(1,2 ; 3 ; 4)$ is

$$
\begin{aligned}
\left.A_{2,1,1}(1,2 ; 3 ; 4)\right|_{\text {cut }}= & A_{L}\left(\ell_{1}, \alpha_{1}, \alpha_{2}, \ell_{2}, \ell_{3}\right) A_{R}\left(-\ell_{1}, \beta_{3},-\ell_{3}, \beta_{4},-\ell_{2}\right) \\
& +A_{L}\left(\ell_{1}, 1, \ell_{2}, \ell_{3}, 3\right) A_{R}\left(-\ell_{1},-\ell_{3}, 4,-\ell_{2}, 2\right)+A_{L}\left(\ell_{1}, 1, \ell_{2}, 3, \ell_{3}\right) A_{R}\left(-\ell_{1}, 4,-\ell_{3},-\ell_{2}, 2\right) \\
& +A_{L}\left(\ell_{1}, 1, \ell_{2}, \ell_{3}, 4\right) A_{R}\left(-\ell_{1},-\ell_{3}, 3,-\ell_{2}, 2\right)+A_{L}\left(\ell_{1}, 1, \ell_{2}, 4, \ell_{3}\right) A_{R}\left(-\ell_{1}, 3,-\ell_{3},-\ell_{2}, 2\right) \\
& +P\left\{\ell_{1}, \ell_{2}, \ell_{3}\right\}
\end{aligned}
$$

where $\alpha, \beta \in Z_{2}$. With these triple cut expressions, we can check identities obtained from $U$ (1)-decoupling equations. An example is given in the Appendix B.

Having above settings, let us study the first question by taking $A_{2,1,1}(1,2 ; 3 ; 4)$ as an example. We want to express this amplitude as ${ }^{13}$

$$
\begin{aligned}
A_{2,1,1}(1,2 ; 3 ; 4)= & x_{1} A_{4}^{L C}(1,2,3,4)+x_{2} A_{4}^{L C}(1,2,4,3)+x_{3} A_{4}^{L C}(2,1,3,4) \\
& +x_{4} A_{4}^{L C}(2,1,4,3)+x_{5} A_{4}^{L C}(1,3,2,4)+x_{6} A_{4}^{L C}(1,4,2,3) .
\end{aligned}
$$

Since $A_{2,1,1}(1,2 ; 3 ; 4)$ is symmetric under $1 \leftrightarrow 2$ and $3 \leftrightarrow 4$, we have $x_{1}=x_{3}, x_{2}=x_{4}, x_{5}=x_{6}$ and $x_{1}=x_{2}$, $x_{3}=x_{4}, x_{5}=x_{6}$. Furthermore, we know that $A_{2,1,1}(1,2 ; 3 ; 4)=A_{1,1,2}(1 ; 2 ; 3,4)$, thus the exchanging of $(1,2) \leftrightarrow(3,4)$ is also symmetric, this tells us $x_{2}=x_{3}$ and $x_{5}=x_{6}$. Putting all these together we get

$$
\begin{aligned}
A_{2,1,1}(1,2 ; 3 ; 4)= & x\left(A_{4}^{L C}(1,2,3,4)+A_{4}^{L C}(1,2,4,3)+A_{4}^{L C}(2,1,3,4)+A_{4}^{L C}(2,1,4,3)\right) \\
& +y\left(A_{4}^{L C}(1,3,2,4)+A_{4}^{L C}(1,4,2,3)\right) .
\end{aligned}
$$

\footnotetext{
${ }^{13}$ We have not assumed any relations between these six amplitudes except the cyclic symmetry. There could be relations and in fact, they do have ones as given in (3.4), but it will not affect the discussion here.
} 
Then the question becomes to find a solution $x, y$ for $(3.19)$. If identity (3.19) is true, it will be true under unitarity cut. Writing down the cut expression as given in, for example, (B.1) and (3.15), at both sides and comparing them, we could obtain equations for $x, y$. If there is nonzero solution of $x, y$ to match up for all cuts, then there is a relation, but if not, then $A_{2,1,1}$ can not be expressed by $A_{4}^{L C}$.

Now we try to solve $x, y$ using the cut $s_{12}$. The contribution for cut $s_{12}$ of $A_{2,1,1}(1,2 ; 3 ; 4)$ has been given in (B.1). Let us use KK relation to take following six amplitudes as basis for the left tree amplitudes:

$$
\begin{array}{lll}
I_{1}=A_{L}\left(\ell_{1}, 1,2, \ell_{2}, \ell_{3}\right), & I_{2}=A_{L}\left(\ell_{1}, 2,1, \ell_{2}, \ell_{3}\right), & I_{3}=A_{L}\left(\ell_{1}, \ell_{2}, 1,2, \ell_{3}\right), \\
I_{4}=A_{L}\left(\ell_{1}, \ell_{2}, 2,1, \ell_{3}\right), & I_{5}=A_{L}\left(\ell_{1}, 1, \ell_{2}, 2, \ell_{3}\right), & I_{6}=A_{L}\left(\ell_{1}, 2, \ell_{2}, 1, \ell_{3}\right),
\end{array}
$$

and another six basis for the right tree amplitudes:

$$
\begin{aligned}
& K_{1}=A_{L}\left(\ell_{1}, 3,4, \ell_{2}, \ell_{3}\right), \quad K_{2}=A_{L}\left(\ell_{1}, 4,3, \ell_{2}, \ell_{3}\right), \quad K_{3}=A_{L}\left(\ell_{1}, \ell_{2}, 3,4, \ell_{3}\right), \\
& K_{4}=A_{L}\left(\ell_{1}, \ell_{2}, 4,3, \ell_{3}\right), \quad K_{5}=A_{L}\left(\ell_{1}, 3, \ell_{2}, 4, \ell_{3}\right), \quad K_{6}=A_{L}\left(\ell_{1}, 4, \ell_{2}, 3, \ell_{3}\right) .
\end{aligned}
$$

Then the coefficients of these $6 \times 6$ basis for the left hand side of (3.19) are given by

$$
\begin{aligned}
& \left(I_{1}+I_{2}\right) \times\left(K_{3}+K_{4}\right) \rightarrow-4, \\
& \left(I_{3}+I_{4}\right) \times\left(K_{1}+K_{2}+K_{3}+K_{4}+K_{5}+K_{6}\right) \rightarrow-4, \\
& \left(I_{5}+I_{6}\right) \times\left(K_{1}+K_{2}+K_{3}+K_{4}+K_{5}+K_{6}\right) \rightarrow-2 .
\end{aligned}
$$

For the right hand side of (3.19), amplitudes $A_{4}^{L C}(1,2,3,4), A_{4}^{L C}(1,2,4,3), A_{4}^{L C}(2,1,3,4)$ and $A_{4}^{L C}(2,1,4,1)$ will contribute to $s_{12}$ cut while $A_{4}^{L C}(1,3,2,4)$ and $A_{4}^{L C}(1,4,2,3)$ will not. By expressing $A_{L}$ and $A_{R}$ with basis $I_{i}, K_{i}$, we get coefficients of these $6 \times 6$ basis as

$$
\begin{aligned}
& \left(I_{1}+I_{2}\right) \times\left(K_{1}+K_{2}\right) \rightarrow 4 x, \\
& \left(I_{1}+I_{2}\right) \times\left(K_{3}+K_{4}+K_{5}+K_{6}\right) \rightarrow 2 x, \\
& \left(I_{3}+I_{4}\right) \times\left(K_{1}+K_{2}+K_{5}+K_{6}\right) \rightarrow 2 x, \\
& \left(I_{5}+I_{6}\right) \times\left(K_{1}+K_{2}\right) \rightarrow 2 x .
\end{aligned}
$$

Comparing above two results it is obviously impossible to find solution $x$, because even the basis at both sides do not match up!

Thus we have our first conclusion: we can not express the double and triple trace partial amplitudes by leading single trace partial amplitudes. Although we have only done the four-point case, we believe the conclusion is true for any $n$. Also we believe it is true for higher loops more than two.

There is one important point we want to remark. In our argument, we have used the KK relation, but not the BCJ relation for tree-level amplitudes, thus our conclusion is true only up to this level. The reason we do not use BCJ relation is that in BCJ relation, the kinematical factors involving $\ell_{i}$ will generally 
appear, thus by unitarity cut method, coefficients of basis (we have assumed there is one basis) will not be related to each other in simple way and we will lose the predicability. We will come back to this point in conclusion section.

Now we move to the second question. From $U(1)$-decoupling method we know that without considering partial amplitudes of subleading-color single trace, we can express all the other partial amplitudes as a linear combination of three independent $A_{4}^{L C}$ and two $A_{2,2}$ (or $A_{2,1,1}$ ). To check if they are really independent, we wish to find a solution of $(\alpha, \beta, x, y, z)$ so that

$$
\alpha A_{2,2}(1,3 ; 2,4)+\beta A_{2,2}(1,4 ; 2,3)=x A_{4}^{L C}(1,2,3,4)+y A_{4}^{L C}(1,2,4,3)+z A_{4}^{L C}(1,3,2,4) .
$$

Let us focus on $s_{12}$ cut, and expand $A_{L}$ and $A_{R}$ in the above basis $I_{i}, K_{i}$. The $A_{4}^{L C}(1,3,2,4)$ 's in right hand side of (3.22) do not contribute to $s_{12}$ cut. The coefficients of $6 \times 6$ basis for the left hand side of (3.22) are

$$
\begin{aligned}
& \left(I_{1}+I_{2}+I_{3}+I_{4}\right) \times\left(K_{1}+K_{2}\right) \rightarrow-4(\alpha+\beta), \quad\left(I_{1}+I_{2}+I_{3}+I_{4}\right) \times\left(K_{5}+K_{6}\right) \rightarrow-2(\alpha+\beta), \\
& \left(I_{5}+I_{6}\right) \times\left(K_{1}+K_{2}+K_{3}+K_{4}\right) \rightarrow-2(\alpha+\beta), \quad I_{5} \times K_{5}=I_{6} \times K_{6}=-6 \alpha, \\
& I_{5} \times K_{6}=I_{6} \times K_{5}=-6 \beta,
\end{aligned}
$$

while the coefficients for the right hand side of (3.22) are

$$
\begin{aligned}
& I_{1} \times K_{1} \rightarrow-2 y, \quad I_{1} \times K_{2} \rightarrow-2 x, \quad I_{1} \times\left(K_{3}+K_{5}\right) \rightarrow-y, \quad I_{1} \times\left(K_{4}+K_{6}\right) \rightarrow-x, \\
& I_{2} \times K_{1} \rightarrow-2 x, \quad I_{2} \times K_{2} \rightarrow-2 y, \quad I_{2} \times\left(K_{3}+K_{5}\right) \rightarrow-x, \quad I_{2} \times\left(K_{4}+K_{6}\right) \rightarrow-y, \\
& I_{3} \times K_{4} \rightarrow-2 x, \quad I_{3} \times K_{3} \rightarrow-2 y, \quad I_{3} \times\left(K_{2}+K_{6}\right) \rightarrow-x, \quad I_{3} \times\left(K_{1}+K_{5}\right) \rightarrow-y, \\
& I_{4} \times K_{3} \rightarrow-2 x, \quad I_{4} \times K_{4} \rightarrow-2 y, \quad I_{4} \times\left(K_{1}+K_{5}\right) \rightarrow-x, \quad I_{4} \times\left(K_{2}+K_{6}\right) \rightarrow-y, \\
& I_{5} \times\left(K_{2}+K_{4}+K_{6}\right) \rightarrow-x, \quad I_{5} \times\left(K_{1}+K_{3}+K_{5}\right) \rightarrow-y, \\
& I_{6} \times\left(K_{2}+K_{4}+K_{6}\right) \rightarrow-y, \quad I_{6} \times\left(K_{1}+K_{3}+K_{5}\right) \rightarrow-x .
\end{aligned}
$$

All these basis should match up for a solution $(\alpha, \beta, x, y, z)$. However, noticing that there are no $I_{1} \times K_{3}$ and $I_{1} \times K_{4}$ terms in left hand side, it gives $x=y=0$, which leads further to $\alpha=\beta=0$. From this argument we see that there is no more relation among three independent $A_{4}^{L C}$ and two $A_{2,2}$ (or $A_{1,1,2}$ ). All these five partial amplitudes are indeed independent to each other.

\subsection{KK-like relation for partial amplitudes of subleading-color single trace}

We have remarked in (3.7) that the $U(1)$-decoupling relation for $A_{4}^{S C}$ is exactly the same as the one for tree-level amplitudes. For general $n$-point $A_{n}^{S C}$, we can also get the same $U(1)$-decoupling relation using $U(1)$-decoupling method

$$
\sum_{\sigma \in \text { cyclic }} A_{n}^{S C}\left(\sigma_{1}, \sigma_{2}, \ldots, \sigma_{n-1}, n\right)=0
$$


where $T^{n}$ has been set to be $U(1)$. This similarity intrigues us to ask if there is KK-like relation for $A_{n}^{S C}$. If the KK relation is true for $A_{n}^{S C}$, the independent partial amplitudes of subleading-color trace will be greatly reduced from $(n-1)$ ! to $(n-2)$ !. Since KK relation can not be derived from $U(1)$-decoupling method, we need to investigate this problem by unitarity cut method.

It is worth to mention that the reflection identity and $U(1)$-decoupling identity, which have been shown to be true, are special cases of KK relation. The first non-trivial KK relation, i.e., KK relation that is different from $U(1)$-decoupling and reflection relation, appears in six-point case. For example, we can write down

$$
\begin{aligned}
A_{6}^{S C}(1,2,3,6,4,5)= & A_{6}^{S C}(1,2,3,5,4,6)+A_{6}^{S C}(1,2,5,3,4,6)+A_{6}^{S C}(1,2,5,4,3,6) \\
& +A_{6}^{S C}(1,5,2,3,4,6)+A_{6}^{S C}(1,5,2,4,3,6)+A_{6}^{S C}(1,5,4,2,3,6) .
\end{aligned}
$$

If above relation is true, it should be true for every triple cut. At first sight it seems obscure, since all terms under the triple cut will have the patterns

$$
A_{L}\left(\ell_{1}, \ldots, \ell_{2}, \ldots, \ell_{3}\right) A_{R}\left(-\ell_{1}, \ldots,-\ell_{2}, \ldots,-\ell_{3}\right), \quad A_{L}\left(\ell_{1}, \ldots, \ell_{3}, \ldots, \ell_{2}\right) A_{R}\left(-\ell_{1}, \ldots,-\ell_{3}, \ldots,-\ell_{2}\right)
$$

which are hard to observe relations among them. The matching of every cut in left and right hand sides of (3.24) is quite non-trivial.

There are totally twenty-five different $\operatorname{cuts}^{14} s_{1 i}, s_{1 i j}$ and $s_{1 i j k}$ for (3.24): fifteen two-particle cuts and ten three-particle cuts. Equation (3.24) has symmetries $\{2 \leftrightarrow 5,3 \leftrightarrow 4\}$ and $\{1 \leftrightarrow 6,4 \leftrightarrow 2,5 \leftrightarrow 3\}$, thus many cuts can be related to each other and we need to check only one cut for each orbit given by symmetry group. With this consideration, cuts to be checked are reduced to the following eleven: six two-particle cuts

$$
s_{12} \sim s_{15} \sim s_{46} \sim s_{36} ; \quad s_{13} \sim s_{14} \sim s_{56} \sim s_{26} ; \quad s_{45} \sim s_{23} ; \quad s_{34} \sim s_{25} ; \quad s_{35} \sim s_{24} ; \quad s_{16},
$$

and five three-particle cuts

$$
s_{126} \sim s_{156} \sim s_{136} \sim s_{146} ; \quad s_{123} \sim s_{145} ; \quad s_{124} \sim s_{135} ; \quad s_{125} ; \quad s_{134}
$$

As an example of how terms match up, we consider cut $s_{134}$, where the expression for $\left.A_{6}^{S C}\right|_{\text {cut }}$ is relatively simple. For the left hand side of (3.24), we have

$$
\left.A_{6}^{S C}(1,2,3,6,4,5)\right|_{c u t}=A_{L}\left(\ell_{1}, 1, \ell_{2}, 4, \ell_{3}, 3\right) A_{R}\left(-\ell_{1}, 6,-\ell_{2}, 2,-\ell_{3}, 5\right)+P\left\{\ell_{1}, \ell_{2}, \ell_{3}\right\} .
$$

\footnotetext{
${ }^{14}$ For general $n$, there are $2^{n-1}-(n+1)$ different cuts to be considered.
} 
For the right hand side, six $\left.A_{6}^{S C}\right|_{\text {cut }}$ 's have following contributions:

$$
\begin{aligned}
& \left.A_{6}^{S C}(1,2,3,5,4,6)\right|_{c u t}=A_{L}\left(\ell_{1}, 1, \ell_{2}, 4, \ell_{3}, 3\right) A_{R}\left(-\ell_{1}, 5,-\ell_{2}, 2,-\ell_{3}, 6\right)+P\left\{\ell_{1}, \ell_{2}, \ell_{3}\right\} \\
& \left.A_{6}^{S C}(1,2,5,3,4,6)\right|_{c u t}= \\
& A_{L}\left(\ell_{1}, 1, \ell_{2}, 3,4, \ell_{3}\right)\left(A_{R}\left(-\ell_{1}, 2,5,-\ell_{2},-\ell_{3}, 6\right)+A_{R}\left(-\ell_{1},-\ell_{2}, 2,5,-\ell_{3}, 6\right)+A_{R}\left(-\ell_{1}, 5,-\ell_{2}, 2,-\ell_{3}, 6\right)\right) \\
& +A_{L}\left(\ell_{1}, 1, \ell_{2}, \ell_{3}, 3,4\right)\left(A_{R}\left(-\ell_{1}, 6,-\ell_{2}, 2,5,-\ell_{3}\right)+A_{R}\left(-\ell_{1},-\ell_{2}, 2,5,-\ell_{3}, 6\right)\right) \\
& \left.+A_{L}\left(\ell_{1}, 1, \ell_{2}, 4, \ell_{3}, 3\right) A_{R}\left(-\ell_{1},-\ell_{2}, 2,5,-\ell_{3}, 6\right)+P\left\{\ell_{1}, \ell_{2}, \ell_{3}\right\}\right)
\end{aligned}
$$

and remaining four partial amplitudes have

$$
\begin{aligned}
& \left.A_{6}^{S C}(1,2,5,4,3,6)\right|_{\text {cut }}=\left.\left(\left.A_{6}^{S C}(1,2,5,3,4,6)\right|_{\text {cut }}\right)\right|_{3 \leftrightarrow 4} ; \\
& \left.A_{6}^{S C}(1,5,2,4,3,6)\right|_{\text {cut }}=\left.\left(\left.A_{6}^{S C}(1,2,5,3,4,6)\right|_{\text {cut }}\right)\right|_{(3 \leftrightarrow 4,2 \leftrightarrow 5)} ; \\
& \left.A_{6}^{S C}(1,5,2,3,4,6)\right|_{\text {cut }}=\left.\left(\left.A_{6}^{S C}(1,2,5,3,4,6)\right|_{\text {cut }}\right)\right|_{2 \leftrightarrow 5} ; \\
& \left.A_{6}^{S C}(1,5,4,2,3,6)\right|_{\text {cut }}=\left.\left(\left.A_{6}^{S C}(1,2,3,5,4,6)\right|_{\text {cut }}\right)\right|_{(3 \leftrightarrow 4,2 \leftrightarrow 5)} .
\end{aligned}
$$

To compare terms at both sides, we expand them into a chosen basis, i.e., the basis independent to each other up to KK relation. The choice we have made here is that leg 3 and 4 of $A_{L}$-part are at the first and last position respectively, while leg 2 and leg 5 of $A_{R}$-part are at the first and last position respectively. Thus we need to compare coefficients of $24 \times 24$ basis at both sides.

In order to make an impression of how these basis match up, we give some details. When we expand six $A^{S C}$ 's at the right hand side of (3.24) by the basis, we will get following expression

$$
\begin{aligned}
& \left.R H S\right|_{c u t}=A_{L}\left(3, \ell_{1}, 1, \ell_{2}, \ell_{3}, 4\right) \times\left(2 A_{R}\left(2,-\ell_{3}, 6,-\ell_{2},-\ell_{1}, 5\right)+2 A_{R}\left(2,-\ell_{3}, 6,-\ell_{1},-\ell_{2}, 5\right)\right. \\
& +A_{R}\left(2,-\ell_{2}, 6,-\ell_{1},-\ell_{3}, 5\right)-A_{R}\left(2,-\ell_{2}, 6,-\ell_{3},-\ell_{1}, 5\right)+A_{R}\left(2,-\ell_{2},-\ell_{3}, 6,-\ell_{1}, 5\right)+A_{R}\left(2,-\ell_{3},-\ell_{2}, 6,-\ell_{1}, 5\right) \\
& \left.+A_{R}\left(2,-\ell_{2},-\ell_{1}, 6,-\ell_{3}, 5\right)+A_{R}\left(2,-\ell_{3},-\ell_{1}, 6,-\ell_{2}, 5\right)-A_{R}\left(2,-\ell_{1},-\ell_{2}, 6,-\ell_{3}, 5\right)+A_{R}\left(2,-\ell_{1},-\ell_{3}, 6,-\ell_{2}, 5\right)\right) \\
& +A_{L}\left(3, \ell_{1}, 1, \ell_{3}, \ell_{2}, 4\right) \times\left(2 A_{R}\left(2,-\ell_{3}, 6,-\ell_{2},-\ell_{1}, 5\right)-A_{R}\left(2,-\ell_{2}, 6,-\ell_{1},-\ell_{3}, 5\right)-A_{R}\left(2,-\ell_{2}, 6,-\ell_{3},-\ell_{1}, 5\right)\right. \\
& +A_{R}\left(2,-\ell_{2},-\ell_{3}, 6,-\ell_{1}, 5\right)+A_{R}\left(2,-\ell_{3},-\ell_{2}, 6,-\ell_{1}, 5\right)-A_{R}\left(2,-\ell_{1},-\ell_{2}, 6,-\ell_{3}, 5\right)+A_{R}\left(2,-\ell_{1},-\ell_{3}, 6,-\ell_{2}, 5\right) \\
& \left.-A_{R}\left(2,-\ell_{2},-\ell_{1}, 6,-\ell_{3}, 5\right)-A_{R}\left(2,-\ell_{3},-\ell_{1}, 6,-\ell_{2}, 5\right)\right)+A_{L}\left(3, \ell_{3}, \ell_{1}, 1, \ell_{2}, 4\right) \times\left(-2 A_{R}\left(2,-\ell_{1}, 6,-\ell_{2},-\ell_{3}, 5\right)\right. \\
& +A_{R}\left(2,-\ell_{2}, 6,-\ell_{3},-\ell_{1}, 5\right)+A_{R}\left(2,-\ell_{2}, 6,-\ell_{1},-\ell_{3}, 5\right)+A_{R}\left(2,-\ell_{3},-\ell_{2}, 6,-\ell_{1}, 5\right)+A_{R}\left(2,-\ell_{2},-\ell_{3}, 6,-\ell_{1}, 5\right) \\
& \left.-2 A_{R}\left(2,-\ell_{1},-\ell_{2}, 6,-\ell_{3}, 5\right)\right)+A_{L}\left(3, \ell_{1}, \ell_{3}, 1, \ell_{2}, 4\right) \times\left(2 A_{R}\left(2,-\ell_{3}, 6,-\ell_{2},-\ell_{1}, 5\right)+A_{R}\left(2,-\ell_{2}, 6,-\ell_{3},-\ell_{1}, 5\right)\right. \\
& \left.+A_{R}\left(2,-\ell_{2}, 6,-\ell_{1},-\ell_{3}, 5\right)+3 A_{R}\left(2,-\ell_{3},-\ell_{2}, 6,-\ell_{1}, 5\right)+A_{R}\left(2,-\ell_{2},-\ell_{3}, 6,-\ell_{1}, 5\right)\right)+P\left\{\ell_{1}, \ell_{2}, \ell_{3}\right\} .
\end{aligned}
$$


Similarly, the expansion of left hand side of (3.24) is equal to following expression

$$
\begin{aligned}
\left.L H S\right|_{\text {cut }}= & \left(A_{L}\left(3, \ell_{1}, 1, \ell_{2}, \ell_{3}, 4\right)+A_{L}\left(3, \ell_{1}, 1, \ell_{3}, \ell_{2}, 4\right)+A_{L}\left(3, \ell_{3}, \ell_{1}, 1, \ell_{2}, 4\right)+A_{L}\left(3, \ell_{1}, \ell_{3}, 1, \ell_{2}, 4\right)\right) \\
& \times\left(A_{R}\left(2,-\ell_{2}, 6,-\ell_{3},-\ell_{1}, 5\right)+A_{R}\left(2,-\ell_{2}, 6,-\ell_{1},-\ell_{3}, 5\right)+A_{R}\left(2,-\ell_{2},-\ell_{3}, 6,-\ell_{1}, 5\right)\right. \\
& \left.+A_{R}\left(2,-\ell_{3},-\ell_{2}, 6,-\ell_{1}, 5\right)\right)+P\left\{\ell_{1}, \ell_{2}, \ell_{3}\right\} .
\end{aligned}
$$

From above expressions, it is very difficult to see that $\left.R H S\right|_{\text {cut }}$ will equal to $\left.L H S\right|_{\text {cut }}$. But if we explicitly write down the terms in $P\left\{\ell_{1}, \ell_{2}, \ell_{3}\right\}$ of $\left.R H S\right|_{c u t}$, we will see much cancellations. For example, if we only explicitly write down the terms with exchange of $\ell_{2}$ and $\ell_{3}$ in $P\left\{\ell_{1}, \ell_{2}, \ell_{3}\right\}$ of $\left.R H S\right|_{\text {cut }}$, the expression will be highly reduced to

$$
\begin{aligned}
& \left.R H S\right|_{\text {cut }}= \\
& \left(A_{L}\left(3, \ell_{1}, 1, \ell_{2}, \ell_{3}, 4\right)+A_{L}\left(3, \ell_{1}, 1, \ell_{3}, \ell_{2}, 4\right)\right) \times\left(2 A_{R}\left(2,-\ell_{2},-\ell_{3}, 6,-\ell_{1}, 5\right)+2 A_{R}\left(2,-\ell_{3},-\ell_{2}, 6,-\ell_{1}, 5\right)\right. \\
& \left.+A_{R}\left(2,-\ell_{3}, 6,-\ell_{2},-\ell_{1}, 5\right)+A_{R}\left(2,-\ell_{3}, 6,-\ell_{1},-\ell_{2}, 5\right)+A_{R}\left(2,-\ell_{2}, 6,-\ell_{3},-\ell_{1}, 5\right)+A_{R}\left(2,-\ell_{2}, 6,-\ell_{1},-\ell_{3}, 5\right)\right) \\
& +\left(A_{L}\left(3, \ell_{3}, \ell_{1}, 1, \ell_{2}, 4\right)+A_{L}\left(3, \ell_{1}, \ell_{3}, 1, \ell_{2}, 4\right)\right) \times\left(2 A_{R}\left(2,-\ell_{2}, 6,-\ell_{3},-\ell_{1}, 5\right)+2 A_{R}\left(2,-\ell_{2}, 6,-\ell_{1},-\ell_{3}, 5\right)\right. \\
& \left.+A_{R}\left(2,-\ell_{2},-\ell_{3}, 6,-\ell_{1}, 5\right)+A_{R}\left(2,-\ell_{3},-\ell_{2}, 6,-\ell_{1}, 5\right)+A_{R}\left(2,-\ell_{2},-\ell_{1}, 6,-\ell_{3}, 5\right)+A_{R}\left(2,-\ell_{1},-\ell_{2}, 6,-\ell_{3}, 5\right)\right) \\
& +C P\left\{\ell_{1}, \ell_{2}, \ell_{3}\right\}
\end{aligned}
$$

which can be easily seen equal to $\left.L H S\right|_{\text {cut }}$.

Unlike the simplicity of the cut $s_{134}$, other cuts are more difficult to check. We have implemented it in Mathematica and found that for all cuts $s_{1 i}, s_{1 i j}$ and $s_{1 i j k}$, after using the KK relation of tree-level amplitudes, the equation (3.24) always holds.

Beyond six-point, we have also checked the case of seven-point by Mathematica and the complexity increases dramatically with the increasing of $n$. For seven points, the KK relation is also true.

Next is the eight-point case, but we found that, by checking several cuts,

$$
A^{S C}(1,\{2,3\}, 8,\{4,5,6,7\}) \neq \sum_{\sigma \in O P\{2,3\} \bigcup\{7,6,5,4\}} A^{S C}(1, \sigma, 8),
$$

where " $\neq "$ means under the cut, the left hand side is not equal to the right hand side. However, we do find that

$$
\begin{aligned}
& A^{S C}(1,\{2,3\}, 8,\{4,5,6,7\})+A^{S C}(1,\{3,2\}, 8,\{4,5,6,7\}) \\
& \sum_{\sigma \in O P\{2,3\} \bigcup\{7,6,5,4\}} A^{S C}(1, \sigma, 8)+\sum_{\sigma \in O P\{3,2\} \bigcup\{7,6,5,4\}} A^{S C}(1, \sigma, 8),
\end{aligned}
$$

where " =" means for all cuts the both sides are equal. For another KK relation of $A^{S C}(1,\{2,3,4\}, 8,\{5,6,7\})$, it is also not true by unitarity cut method, but we find that

$$
\sum_{\text {cyclic }\{2,3,4\}} A^{S C}(1,\{2,3,4\}, 8,\{5,6,7\})=-\sum_{\text {cyclic }\{2,3,4\}} \sum_{\sigma \in O P\{2,3,4\} \bigcup\{7,6,5\}} A^{S C}(1, \sigma, 8)
$$


is true under all triple cuts. It is also strange to find that the relation

$$
\sum_{\text {cyclic }\{2,3,4\}} A^{S C}(1,\{2,3,4,5\}, 8,\{6,7\})=\sum_{\text {cyclic }\{2,3,4\}} \sum_{\sigma \in O P\{2,3,4,5\} \bigcup\{7,6\}} A^{S C}(1, \sigma, 8)
$$

is true under all triple cuts. The case of nine point is too complicated even for the computer.

The observation of eight point is very mysterious for us and we do not understand why naive KK relation fails for higher points. It is possible that KK relation is true for higher points, but our triple cut method can not assure it. In other words, although the integrands at both sides do not match up under our unitarity cut method, the final integrated results may match up. We are continuing the investigation of this problem.

\section{Conclusion}

In this paper we have used the unitarity cut method [1, 2] to study relations among color-ordered partial amplitudes of gauge theory at one-loop and two-loop. At one-loop we have proved the known result (2.2) that partial amplitudes of double trace structure can be completely solved as a linear combination of primitive amplitudes [3] by using KK relation of tree-level amplitudes. Our proof gives a clear physical picture for the similarity between relation (2.2) and tree-level KK relation (1.2). The reflection identity of any-loop amplitudes can also be understood explicitly from reflection identity of tree amplitudes by unitarity cut method although it can also be understood directly from the pure group property of gauge theory.

At two-loop level, unitarity cut method has also helped us to understand several interesting questions. First it is shown that just partial amplitudes of leading-color single trace structure are not enough to solve partial amplitudes of other trace structures. This can also be understood by noticing that leadingcolor partial amplitudes include only planar diagrams ${ }^{15}$. Then the unitarity cut method leads us to the possibility that there is KK-like relation for partial amplitudes of subleading-color single trace structure, where examples of six-, seven- and eight-point, have been explicitly studied.

Our result in this paper is only one little step of the application of unitarity cut method for understanding the relation of loop amplitudes. There are many things which are still not clear and should be discussed in future.

The first thing we want to understand more is the role of tree-level BCJ relation for loop amplitudes. In this paper, we have used only tree-level KK relation and have deliberately avoided the use of BCJ relation. The main reason is that BCJ relation will involve the kinematic factors $s_{\ell_{i} i}$, which makes the discussion in the frame of unitarity cut method very complicated. The generalization of BCJ relation to loop-level has been discussed in [32, 37, 38, 39], where not the whole partial amplitude, but some parts of it have relations. The correspondence of this point in the unitarity cut method is following: we may get match up for some cuts, but not for all cuts. Thus we do not get the relation for the whole amplitude,

\footnotetext{
${ }^{15}$ We would like to thank referee for several enlightening remarks.
} 
but do get relations for parts of amplitude detected by these matching cuts. Of course, many works are needed to make above picture clear.

The second thing worth to do is to systematically study two-loop partial amplitudes. The mysterious KK-like relation for subleading-color single trace partial amplitudes has not been understood. The similarity has also intrigued us to ask the possibility of BCJ-like relation for subleading-color single trace partial amplitudes. Moreover, although the basis found by $U(1)$-decoupling method in four-point case is the same basis found by unitarity cut method, we are not sure if this will be true for general $n$. Just like one-loop example, (2.2) reduces to $U(1)$-decoupling equation for $n \leq 5$, but is different for $n \geq 6$.

It is also interesting to use unitarity cut method to discuss partial amplitudes for more than two loops. With the increasing of loops, the complexity will also increase a lot, thus a better idea to implement this method would be welcome.

\section{Acknowledgements}

We would like to thank Rutger Boels for collaboration at early stage of this project and the hospitality of KITPC, China, where final part of this work was done. We are supported by fund from Qiu-Shi, the Fundamental Research Funds for the Central Universities with contract number 2010QNA3015, as well as Chinese NSF funding under contract No.10875104, No.11031005.

\section{A. Direct verification of relations for two-loop four-point amplitude}

Two-loop four-gluon partial amplitudes of $S U(N) \mathcal{N}=4$ super-Yang-Mills theory have been computed in 443 by cut method. We would like to verify the relations of two-loop four-point amplitudes directly using these results.

The relations we have obtained are

$$
0=A_{4}^{S C}(1,2,3,4)+A_{4}^{S C}(1,2,4,3)+A_{4}^{S C}(1,4,2,3),
$$

and

$$
A_{2,2}(1,2 ; 3,4)+A_{2,2}(1,3 ; 2,4)+A_{2,2}(1,4 ; 2,3)=3 \sum_{\sigma \in S_{4} / Z_{4}} A_{4}^{L C}(\sigma)
$$

In order to verify these two relations, we need to know the corresponding partial amplitudes. In 43. partial amplitudes are given as a linear combination of some planar and non-planar basis. Written in our convention, the leading-color single trace amplitude is

$$
A_{4}^{L C}(1,2,3,4)=A_{4}^{P}(1,2 ; 3,4)+A_{4}^{P}(1,4 ; 3,2),
$$

and the subleading-color single trace amplitude is

$$
\begin{aligned}
A_{4}^{S C}(1,2,3,4)= & 2 A_{4}^{P}(1,2 ; 3,4)+2 A_{4}^{P}(1,2 ; 4,3)+2 A_{4}^{P}(1,4 ; 2,3)+2 A_{4}^{P}(1,4 ; 3,2) \\
& -4 A_{4}^{P}(1,3 ; 2,4)-4 A_{4}^{P}(1,3 ; 4,2)+2 A_{4}^{N P}(1,2 ; 3,4)+2 A_{4}^{N P}(1,2 ; 4,3) \\
& +2 A_{4}^{N P}(1,4 ; 2,3)+2 A_{4}^{N P}(1,4 ; 3,2)-4 A_{4}^{N P}(1,3 ; 2,4)-4 A_{4}^{N P}(1,3 ; 4,2)
\end{aligned}
$$


then the double trace amplitude is

$$
\begin{aligned}
A_{2,2}(1,2 ; 3,4)= & 6 A_{4}^{P}(1,2 ; 3,4)+6 A_{4}^{P}(1,2 ; 4,3)+4 A_{4}^{N P}(1,2 ; 3,4)+4 A_{4}^{N P}(1,2 ; 4,3) \\
& -2 A_{4}^{N P}(1,4 ; 2,3)-2 A_{4}^{N P}(1,4 ; 3,2)-2 A_{4}^{N P}(1,3 ; 2,4)-2 A_{4}^{N P}(1,3 ; 4,2)
\end{aligned}
$$

where $A^{P}$ and $A^{N P}$ are functions of two-loop planar and non-planar scalar double-box integrals as defined in 43 .

Firstly let us verify (A.1). The coefficients of each basis can be directly written down as

\begin{tabular}{c|c|c|c|c|c|c} 
& $A_{4}^{P}(1,2 ; 3,4)$ & $A_{4}^{P}(1,2 ; 4,3)$ & $A_{4}^{P}(1,3 ; 2,4)$ & $A_{4}^{P}(1,3 ; 4,2)$ & $A_{4}^{P}(1,4 ; 2,3)$ & $A_{4}^{P}(1,4 ; 3,2)$ \\
\hline$A_{4}^{S C}(1,2,3,4)$ & 2 & 2 & -4 & -4 & 2 & 2 \\
\hline$A_{4}^{S C}(1,2,4,3)$ & 2 & 2 & 2 & 2 & -4 & -4 \\
\hline$A_{4}^{S C}(1,4,2,3)$ & -4 & -4 & 2 & 2 & 2 & 2
\end{tabular}.

It is clear to see that sum of each basis is zero. The sum for non-planar basis is the same as planar basis, thus verifing (A.1).

Then we continue to verify (A.2). We consider planar basis, and for the left hand side we have

\begin{tabular}{c|c|c|c|c|c|c} 
& $A_{4}^{P}(1,2 ; 3,4)$ & $A_{4}^{P}(1,2 ; 4,3)$ & $A_{4}^{P}(1,3 ; 2,4)$ & $A_{4}^{P}(1,3 ; 4,2)$ & $A_{4}^{P}(1,4 ; 2,3)$ & $A_{4}^{P}(1,4 ; 3,2)$ \\
\hline$A_{2,2}(1,2 ; 3,4)$ & 6 & 6 & 0 & 0 & 0 & 0 \\
\hline$A_{2,2}(1,3 ; 2,4)$ & 0 & 0 & 6 & 6 & 0 & 0 \\
\hline$A_{2,2}(1,4 ; 2,3)$ & 0 & 0 & 0 & 0 & 6 & 6
\end{tabular}.

The coefficient for each two-loop planar basis is six. It is easy to get the coefficient for each basis of the right hand side, which is also six.

Then let us consider non-planar basis, which come from only the left hand side, and we have

\begin{tabular}{c|c|c|c|c|c|c} 
& $A_{4}^{N P}(1,2 ; 3,4)$ & $A_{4}^{N P}(1,2 ; 4,3)$ & $A_{4}^{N P}(1,3 ; 2,4)$ & $A_{4}^{N P}(1,3 ; 4,2)$ & $A_{4}^{N P}(1,4 ; 2,3)$ & $A_{4}^{N P}(1,4 ; 3,2)$ \\
\hline$A_{2,2}(1,2 ; 3,4)$ & 4 & 4 & -2 & -2 & -2 & -2 \\
\hline$A_{2,2}(1,3 ; 2,4)$ & -2 & -2 & 4 & 4 & -2 & -2 \\
\hline$A_{2,2}(1,4 ; 2,3)$ & -2 & -2 & -2 & -2 & 4 & 4
\end{tabular}.

This gives a zero result, thus verifing (A.2).

\section{B. The proof of identity $A_{1,1,2}(1 ; 2 ; 3,4)=A_{2,1,1}(1,2 ; 3 ; 4)$}

To demonstrate the use of triple cut method, we give the proof of $A_{1,1,2}(1 ; 2 ; 3,4)=A_{2,1,1}(1,2 ; 3 ; 4)$. This identity does not directly come from $U(1)$-decoupling equations, but is obtained from solving these $U(1)$-decoupling equations.

Before comparing two sides under various cuts, we need to obtain contributions of a given cut. The contributions in general are given by $A_{L}\left(\ell_{1}, \alpha(1), \ell_{2}, \beta, \ell_{3}, \gamma\right)$ plus permutations $P\left(\ell_{1}, \ell_{2}, \ell_{3}\right)$, where $\alpha(1)$ 
means that leg 1 belongs to set $\alpha$. This is equal to fix $\ell_{1}$ at the first position, but fix leg 1 at the set $\alpha, \beta, \gamma$ plus $\ell_{2} \leftrightarrow \ell_{3}$. Using this convention, we write down contributions for the cut $s_{12}$.

For the trace structure $\operatorname{Tr}(1,2) \operatorname{Tr}(3) \operatorname{Tr}(4)$ we have following terms

$$
\begin{aligned}
& A_{1}=\left[A_{L}\left(\ell_{1}, 1,2, \ell_{2}, \ell_{3}\right)+\{1 \leftrightarrow 2\}\right] \times\left[A_{R}\left(-\ell_{1}, 3,-\ell_{3}, 4,-\ell_{2}\right)+A_{R}\left(-\ell_{1}, 4,-\ell_{3}, 3,-\ell_{2}\right)\right], \\
& A_{2}=\left[A_{L}\left(\ell_{1}, \ell_{2}, 1,2, \ell_{3}\right)+\{1 \leftrightarrow 2\}\right] \times\left[A_{R}\left(-\ell_{1}, 3,-\ell_{3},-\ell_{2}, 4\right)+A_{R}\left(-\ell_{1}, 4,-\ell_{3},-\ell_{2}, 3\right)\right], \\
& A_{3}=\left[A_{L}\left(\ell_{1}, \ell_{2}, \ell_{3}, 1,2\right)+\{1 \leftrightarrow 2\}\right] \times\left[A_{R}\left(-\ell_{1},-\ell_{3}, 3,-\ell_{2}, 4\right)+A_{R}\left(-\ell_{1},-\ell_{3}, 4,-\ell_{2}, 3\right)\right], \\
& A_{4}=A_{1}\left(\left\{\ell_{2} \leftrightarrow \ell_{3}\right\}\right), \quad A_{5}=A_{2}\left(\left\{\ell_{2} \leftrightarrow \ell_{3}\right\}\right), \quad A_{6}=A_{3}\left(\left\{\ell_{2} \leftrightarrow \ell_{3}\right\}\right),
\end{aligned}
$$

while for the trace structure $\operatorname{Tr}(1) \operatorname{Tr}(2) \operatorname{Tr}(3,4)$ we have following terms

$$
\begin{aligned}
& B_{1}=\left[A_{L}\left(\ell_{1}, 1, \ell_{2}, 2, \ell_{3}\right)+A_{L}\left(\ell_{1}, 2, \ell_{2}, 1, \ell_{3}\right)\right] \times\left[A_{R}\left(-\ell_{1}, 3,4,-\ell_{3},-\ell_{2}\right)+\{3 \leftrightarrow 4\}\right], \\
& B_{2}=\left[A_{L}\left(\ell_{1}, 1, \ell_{2}, \ell_{3}, 2\right)+A_{L}\left(\ell_{1}, 2, \ell_{2}, \ell_{3}, 1\right)\right] \times\left[A_{R}\left(-\ell_{1},-\ell_{3}, 3,4,-\ell_{2}\right)+\{3 \leftrightarrow 4\}\right], \\
& B_{3}=\left[A_{L}\left(\ell_{1}, \ell_{2}, 1, \ell_{3}, 2\right)+A_{L}\left(\ell_{1}, \ell_{2}, 2, \ell_{3}, 1\right)\right] \times\left[A_{R}\left(-\ell_{1},-\ell_{3},-\ell_{2}, 3,4\right)+\{3 \leftrightarrow 4\}\right], \\
& B_{4}=B_{1}\left(\left\{\ell_{2} \leftrightarrow \ell_{3}\right\}\right), \quad B_{5}=B_{2}\left(\left\{\ell_{2} \leftrightarrow \ell_{3}\right\}\right), \quad B_{6}=B_{3}\left(\left\{\ell_{2} \leftrightarrow \ell_{3}\right\}\right) .
\end{aligned}
$$

To show the equality, we rewrite

$$
\begin{aligned}
& -2\left[A_{L}\left(\ell_{1}, 1,2, \ell_{2}, \ell_{3}\right)+A_{L}\left(\ell_{1}, 2,1, \ell_{2}, \ell_{3}\right)\right]=A_{L}\left(\ell_{1}, 1, \ell_{2}, 2, \ell_{3}\right)+A_{L}\left(\ell_{1}, 1, \ell_{2}, \ell_{3}, 2\right) \\
& +A_{L}\left(\ell_{1}, 2, \ell_{2}, 1, \ell_{3}\right)+A_{L}\left(\ell_{1}, 2, \ell_{2}, \ell_{3}, 1\right)
\end{aligned}
$$

so the ordering with 1,2 nearby in (B.1) is transferred to the ordering with 1,2 not nearby as given in (B.2). Similarly using

$$
\begin{aligned}
& -2\left[A_{R}\left(-\ell_{1}, 3,4,-\ell_{2},-\ell_{3}\right)+A_{R}\left(-\ell_{1}, 4,3,-\ell_{2},-\ell_{3}\right)\right]=A_{R}\left(-\ell_{1}, 3,-\ell_{2}, 4,-\ell_{3}\right)+A_{R}\left(-\ell_{1}, 3,-\ell_{2},-\ell_{3}, 4\right) \\
& +A_{R}\left(-\ell_{1}, 4,-\ell_{2}, 3,-\ell_{3}\right)+A_{R}\left(-\ell_{1}, 4,-\ell_{2},-\ell_{3}, 3\right)
\end{aligned}
$$

the form in (B.2) will become the form in (B.1). Then we just need to put (B.3) back to (B.1) and (B.4) back to $(\mathrm{B} .2)$, and compare the terms in $A_{i}$ and $B_{i}$. For example, the term with ordering $\left(\ell_{1}, 1, \ell_{2}, 2, \ell_{3}\right)$ coming from $A_{1}$ and $A_{2}$ will be multiplied with

$$
\frac{-1}{2}\left[A_{R}\left(-\ell_{1}, 3,-\ell_{3}, 4,-\ell_{2}\right)+A_{R}\left(-\ell_{1}, 3,-\ell_{3},-\ell_{2}, 4\right)+\{3 \leftrightarrow 4\}\right],
$$

while the term with ordering $\left(\ell_{1}, 1, \ell_{2}, 2, \ell_{3}\right)$ only coming from $B_{1}$ will be multiplied with

$$
A_{R}\left(-\ell_{1}, 3,4,-\ell_{3},-\ell_{2}\right)+A_{R}\left(-\ell_{1}, 4,3,-\ell_{3},-\ell_{2}\right)
$$

which is nothing but (B.5) according to (B.4). Other terms can easily be checked using the same procedure.

Cut $s_{13}$ and $s_{14}$ can be checked in the similar way, thus we prove the identity by unitarity cut method. 


\section{References}

[1] L. D. Landau, Nucl. Phys. 13, 181 (1959);

S. Mandelstam, Phys. Rev. 112, 1344 (1958);

S. Mandelstam, Phys. Rev. 115, 1741 (1959);

R. E. Cutkosky, J. Math. Phys. 1, 429 (1960).

[2] Z. Bern, L. J. Dixon, D. C. Dunbar and D. A. Kosower, Nucl. Phys. B 435, 59 (1995) [arXiv:hep-ph/9409265].

[3] Z. Bern, L. J. Dixon, D. C. Dunbar and D. A. Kosower, Nucl. Phys. B 425, 217 (1994)

[arXiv:hep-ph/9403226].

[4] R. Britto, F. Cachazo and B. Feng, Nucl. Phys. B 725, 275 (2005) [arXiv:hep-th/0412103].

[5] R. Britto, E. Buchbinder, F. Cachazo and B. Feng, Phys. Rev. D 72, 065012 (2005) [arXiv:hep-ph/0503132].

[6] C. Anastasiou, R. Britto, B. Feng, Z. Kunszt and P. Mastrolia, Phys. Lett. B 645, 213 (2007) [arXiv:hep-ph/0609191]. C. Anastasiou, R. Britto, B. Feng, Z. Kunszt and P. Mastrolia, JHEP 0703, 111 (2007) [arXiv:hep-ph/0612277].

[7] F. Cachazo, P. Svrcek and E. Witten, JHEP 0409, 006 (2004) [arXiv:hep-th/0403047].

[8] E. Witten, Commun. Math. Phys. 252, 189 (2004) [arXiv:hep-th/0312171].

[9] R. Britto, F. Cachazo and B. Feng, Nucl. Phys. B 715, 499 (2005) [arXiv:hep-th/0412308];

[10] R. Britto, F. Cachazo, B. Feng and E. Witten, Phys. Rev. Lett. 94, 181602 (2005) [arXiv:hep-th/0501052].

[11] M. L. Mangano, S. J. Parke and Z. Xu, Nucl. Phys. B 298, 653 (1988).

[12] Z. Bern and D. A. Kosower, Nucl. Phys. B 362, 389 (1991).

[13] M. L. Mangano and S. J. Parke, Phys. Rept. 200, 301 (1991) [arXiv:hep-th/0509223].

[14] R. Kleiss and H. Kuijf, Nucl. Phys. B 312, 616 (1989).

[15] V. Del Duca, L. J. Dixon and F. Maltoni, Nucl. Phys. B 571, 51 (2000) [arXiv:hep-ph/9910563].

[16] N. E. J. Bjerrum-Bohr, P. H. Damgaard and P. Vanhove, Phys. Rev. Lett. 103, 161602 (2009) [arXiv:0907.1425 [hep-th]].

[17] S. Stieberger, arXiv:0907.2211 [hep-th].

[18] Z. Bern, J. J. M. Carrasco and H. Johansson, Phys. Rev. D 78, 085011 (2008) [arXiv:0805.3993 [hep-ph]].

[19] B. Feng, R. Huang and Y. Jia, arXiv:1004.3417 [hep-th].

[20] Y. X. Chen, Y. J. Du and B. Feng, JHEP 1102, 112 (2011) [arXiv:1101.0009 [hep-th]].

[21] Y. Jia, R. Huang and C. Y. Liu, Phys. Rev. D 82, 065001 (2010) [arXiv:1005.1821 [hep-th]].

[22] J. H. Huang, R. Huang and Y. Jia, arXiv:1009.5073 [hep-th].

[23] H. Zhang, arXiv:1005.4462 [hep-th].

[24] F. A. Berends and W. Giele, Nucl. Phys. B 294, 700 (1987). 
[25] M. L. Mangano, Nucl. Phys. B 309, 461 (1988).

[26] L. J. Dixon, arXiv:hep-ph/9601359.

[27] T. Sondergaard, Nucl. Phys. B 821, 417 (2009) [arXiv:0903.5453 [hep-th]].

[28] S. H. Henry Tye and Y. Zhang, JHEP 1006, 071 (2010) [arXiv:1003.1732 [hep-th]].

[29] N. E. J. Bjerrum-Bohr, P. H. Damgaard, T. Sondergaard and P. Vanhove, JHEP 1006, 003 (2010) [arXiv:1003.2403 [hep-th]].

[30] C. R. Mafra, JHEP 1001, 007 (2010) [arXiv:0909.5206 [hep-th]].

[31] C. R. Mafra, O. Schlotterer, S. Stieberger and D. Tsimpis, arXiv:1012.3981 [hep-th].

[32] N. E. J. Bjerrum-Bohr, P. H. Damgaard, H. Johansson and T. Sondergaard, arXiv:1103.6190 [hep-th].

[33] E. I. Buchbinder and F. Cachazo, JHEP 0511, 036 (2005) [arXiv:hep-th/0506126].

[34] F. Cachazo, arXiv:0803.1988 [hep-th].

[35] F. Cachazo, M. Spradlin and A. Volovich, Phys. Rev. D 78, 105022 (2008) [arXiv:0805.4832 [hep-th]].

[36] N. Arkani-Hamed, F. Cachazo, C. Cheung and J. Kaplan, JHEP 1003, 020 (2010) [arXiv:0907.5418 [hep-th]].

[37] Z. Bern, J. J. M. Carrasco and H. Johansson, Phys. Rev. Lett. 105, 061602 (2010) [arXiv:1004.0476 [hep-th]].

[38] Z. Bern, T. Dennen, Y. t. Huang and M. Kiermaier, Phys. Rev. D 82, 065003 (2010) [arXiv:1004.0693 [hep-th]].

[39] Z. Bern and T. Dennen, arXiv:1103.0312 [hep-th].

[40] J. J. M. Carrasco and H. Johansson, arXiv:1103.3298 [hep-th].

[41] L.M. Brown and R.P. Feynman, Phys. Rev. 85:231 (1952);

G. Passarino and M. Veltman, Nucl. Phys. B160:151 (1979);

G. 't Hooft and M. Veltman, Nucl. Phys. B153:365 (1979);

R. G. Stuart, Comp. Phys. Comm. 48:367 (1988);

R. G. Stuart and A. Gongora, Comp. Phys. Comm. 56:337 (1990).

[42] J. Gluza, K. Kajda and D. A. Kosower, Phys. Rev. D 83, 045012 (2011) [arXiv:1009.0472 [hep-th]].

[43] Z. Bern, J. S. Rozowsky and B. Yan, Phys. Lett. B 401, 273 (1997) [arXiv:hep-ph/9702424].

[44] M. x. Luo and C. k. Wen, JHEP 0411, 004 (2004) [arXiv:hep-th/0410045].

[45] M. x. Luo and C. k. Wen, Phys. Lett. B 609, 86 (2005) [arXiv:hep-th/0410118].

[46] Z. Bern, L. J. Dixon and D. A. Kosower, JHEP 0001, 027 (2000) [arXiv:hep-ph/0001001].

[47] Z. Bern, L. J. Dixon, D. A. Kosower, R. Roiban, M. Spradlin, C. Vergu and A. Volovich, Phys. Rev. D 78, 045007 (2008) [arXiv:0803.1465 [hep-th]].

[48] J. M. Drummond and J. M. Henn, JHEP 1105, 105 (2011) [arXiv:1008.2965 [hep-th]].

[49] J. Gluza, K. Kajda and D. A. Kosower, Phys. Rev. D 83, 045012 (2011) [arXiv:1009.0472 [hep-th]]. 
[50] Z. Bern, A. De Freitas and L. J. Dixon, JHEP 0203, 018 (2002) [arXiv:hep-ph/0201161].

[51] Z. Bern, J. J. M. Carrasco, L. J. Dixon, H. Johansson and R. Roiban, Phys. Rev. D 82, 125040 (2010) [arXiv:1008.3327 [hep-th]]. 